\title{
DEGREES OF UNSOLVABILITY OF CONTINUOUS FUNCTIONS
}

JOSEPH S. MILLER

\begin{abstract}
We show that the Turing degrees are not sufficient to measure the complexity of continuous functions on $[0,1]$. Computability of continuous real functions is a standard notion from computable analysis. However, no satisfactory theory of degrees of continuous functions exists. We introduce the continuous degrees and prove that they are a proper extension of the Turing degrees and a proper substructure of the enumeration degrees. Call continuous degrees which are not Turing degrees non-total. Several fundamental results are proved: a continuous function with non-total degree has no least degree representation, settling a question asked by Pour-El and Lempp; every non-computable $f \in \mathcal{C}[0,1]$ computes a non-computable subset of $\mathbb{N}$; there is a non-total degree between Turing degrees $\mathbf{a}<_{T} \mathbf{b}$ iff $\mathbf{b}$ is a PA degree relative to $\mathbf{a} ; \mathcal{S} \subseteq 2^{\mathbb{N}}$ is a Scott set iff it is the collection of $f$-computable subsets of $\mathbb{N}$ for some $f \in \mathcal{C}[0,1]$ of non-total degree; and there are computably incomparable $f, g \in \mathcal{C}[0,1]$ which compute exactly the same subsets of $\mathbb{N}$. Proofs draw from classical analysis and constructive analysis as well as from computability theory.
\end{abstract}

§1. Introduction. The computable real numbers were introduced in Alan Turing's famous 1936 paper, "On computable numbers, with an application to the Entscheidungsproblem" [40]. Originally, they were defined to be the reals with computable decimal expansions, though in 1937 Turing suggested an alternative representation, "modifying the manner in which computable numbers are associated with computable sequences, the totality of computable numbers being left unaltered" [41]. His second representation avoids the problem of nonuniformity at rationals which have finite decimal expansions and is suitable for studying computable functions of reals, though he did not do so. Turing associates the real number $\left(2 c_{0}-1\right) n+\sum_{r=1}^{\infty}\left(2 c_{r}-1\right)(2 / 3)^{r}$ to the infinite binary sequence $c_{0} 1^{n} 0 c_{1} c_{2} \cdots \in\{0,1\}^{\omega}{ }^{1}$. Our choice of representation differs from Turing's, but it is equivalent in the sense of Kreitz and Weihrauch [15]; in particular, they both induce the same computable structure on $\mathbb{R}$. See Weihrauch [43] for a thorough introduction to the theory of representations. ${ }^{2}$

Definition 1.1. A representation of $x \in \mathbb{R}$ is a function $\lambda: \mathbb{Q}^{+} \rightarrow \mathbb{Q}$ such that, for all $\varepsilon \in \mathbb{Q}^{+},|x-\lambda(\varepsilon)|<\varepsilon$.

Assuming that we identify $\mathbb{Q}^{+}$and $\mathbb{Q}$ with effective enumerations of these sets, representations are simply functions from $\mathbb{N}$ to itself, so classical computability

The author's research was partially supported by an NSF VIGRE Fellowship at Indiana University Bloomington.

${ }^{1}$ Turing gives Brouwer credit for the use of overlapping intervals to define real numbers.

${ }^{2}$ Note that Weihrauch uses "representation" to denote a naming system, not an individual name [43]; we abusively use the same word for both. 
theory supplies us with a computable structure on representations. Lifting this structure to $\mathbb{R}$, a real $x \in \mathbb{R}$ is computable if it has a computable representation. Moreover, it is natural to define the Turing degree of $x \in \mathbb{R}$ to be

$$
\operatorname{deg}_{T}(x)=\min \left\{\operatorname{deg}_{T}(\lambda) \mid \lambda \text { represents } x\right\} .
$$

Note that $\operatorname{deg}_{T}(x)$ is well defined for every $x \in \mathbb{R}$ and that it is identical to the Turing degree of the binary expansion of $x$.

One might hope to define the Turing degree of a continuous function $f \in \mathcal{C}[0,1]$ in the same manner. Once we have chosen an appropriate representation for continuous functions - this is done in Section 2 and several equivalent representations can be found in Weihrauch [43]—we would again define

$$
\operatorname{deg}_{T}(f)=\min \left\{\operatorname{deg}_{T}(\lambda) \mid \lambda \text { represents } f\right\},
$$

where $f \in \mathcal{C}[0,1]$. But is the Turing degree of a function always defined? We must answer the following question.

Question 1.2 (Pour-El and Lempp). Does every $f \in \mathcal{C}[0,1]$ have a representation of least Turing degree?

An analogous question was studied in computable model theory by L. J. Richter [29]. A countable group $\mathcal{G}$ can be presented as a subset of $\mathbb{N}$ with a binary relation representing multiplication. Other countable structures, such as linear orders and graphs, can be presented similarly. Just as a function $f \in \mathcal{C}[0,1]$ has infinitely many representations, $\mathcal{G}$ will have infinitely many presentations. Say that $\mathcal{G}$ computes $A \subseteq \mathbb{N}$ if every one of its presentations computes $A$, and that $\mathcal{G}$ has Turing degree $\mathbf{a}$ if this is the least degree of any presentation. Richter proved that there are groups of every Turing degree, but also that there are groups which have no Turing degree. There are even non-computable groups which compute no non-computable subsets of $\mathbb{N}$. The situation for linear orders is more restrictive; no linear order can compute a non-computable subset of $\mathbb{N}$, so in fact, no non-computable linear order has a Turing degree.

Our goal is not only to answer Question 1.2 in the negative, but also to introduce a natural degree structure which captures the complexity of the continuous functions and initiate the study of this structure. Our methods are very different from those used by Richter. The outcome is also different; we not only show that there are continuous functions with no Turing degree, but also that every non-computable $f \in \mathcal{C}[0,1]$ computes a non-computable subset of $\mathbb{N}$, distinguishing the effective content of continuous functions from that of groups and linear orders and from the various other classes of discrete structures that have been studied.

The article is organized as follows. Section 2 is an introduction to computable analysis on computable metric spaces. For our purposes, the most important examples of computable metric spaces are $2^{\mathbb{N}}, \mathbb{N}^{\mathbb{N}}, \mathbb{R}, \mathcal{C}[0,1]$ and $[0,1]^{\mathbb{N}}$; these are discussed briefly. Section 3 defines representation reducibility, a notion of relative computability between elements of computable metric spaces. The induced 
degree structure is called the continuous degrees. Representation reducibility agrees with Turing reducibility on $2^{\mathbb{N}}$ and $\mathbb{N}^{\mathbb{N}}$, so the continuous degrees extend the Turing degrees. Call a continuous degree which corresponds to a Turing degree total. We show that every continuous degree contains elements of $\mathcal{C}[0,1]$, justifying the name, and also of $[0,1]^{\mathbb{N}}$. Section 4 begins with a review of the enumeration degrees (sometimes called the partial degrees). We then show that the continuous degrees embed into the enumeration degrees and observe that the existence of a non-total continuous degree would provide a negative answer to Question 1.2.

The key to the results in later sections is the reduction of questions about degrees of continuous functions to questions about sequences of reals. In Section 5 , we consider sequences in $[0,1]^{\mathbb{N}}$ which list all of the reals in $[0,1]$ which they compute. Such a sequence is not computably diagonalizable. We show that the non-total continuous degrees are exactly the degrees of sequences which are not computably diagonalizable. In Section 6, we construct a sequence which is not computably diagonalizable using a classical topological fixed point theorem for multivalued functions on the Hilbert cube $[0,1]^{\mathbb{N}}$. This proves that non-total continuous degrees exist, which proves that there is a function $f \in \mathcal{C}[0,1]$ with no least Turing degree representation.

Having solved the problem which motivated this research, we begin to investigate the nature of non-total continuous degrees in Section 7. In doing so, we distinguish the continuous degrees from the enumeration degrees and also contrast our results from those of Richter. A modification of Orevkov's constructive retraction of (the constructive points of) the unit square onto its boundary is used to show that every sequence of computable reals is computably diagonalizable. This implies that every non-computable continuous function computes a non-computable subset of $\mathbb{N}$. It also provides us with an elementary difference between the enumeration degrees and the continuous degrees; only the latter has minimal elements.

The last two sections are concerned with the relationship of the continuous degrees to the substructure of the Turing degrees. Classical concepts play an important role. If $\mathbf{a}$ and $\mathbf{b}$ are Turing degrees, we say that $\mathbf{a}$ is a $P A$ degree relative to $\mathbf{b}(\mathbf{b} \ll \mathbf{a})$ if every infinite $\mathbf{b}$-computable subtree of $2^{<\mathbb{N}}$ has an infinite path computable from a. A Scott ideal is a countable ideal in the Turing degrees such that for every $\mathbf{b} \in \mathcal{I}$ there is an $\mathbf{a} \in \mathcal{I}$ with $\mathbf{b} \ll \mathbf{a}$. If $\mathcal{I}$ is a Scott ideal, then the collection of subsets of $\mathbb{N}$ with degree in $\mathcal{I}$ is called a Scott set. PA degrees and Scott sets arose from the study of complete extensions of Peano arithmetic and turn out to be closely connected to the continuous degrees.

The main result of Section 8 is proved by a more careful analysis of the techniques from Sections 6 and 7 . It characterizes the intervals $\mathbf{b} \leq \mathbf{a}$ of Turing degrees which contain a non-total continuous degree as the intervals $\mathbf{b} \ll \mathbf{a}$. This is a significant restriction on the structure of the continuous degrees; using it we prove that the continuous degrees are not a lattice and that the first order 
theory of the continuous degrees is equivalent to second order arithmetic. In addition, we distinguish the first order theories of the continuous degrees and the Turing degrees. Therefore, reducibility between continuous functions really does give rise to a new degree structure. It is also proved in Section 8 that if $\mathbf{b}$ is a Turing degree below a non-total continuous degree $\mathbf{v}$, then there is another Turing degree $\mathbf{c}$ with $\mathbf{b} \ll \mathbf{c}<\mathbf{v}$. This implies that the collection of subsets of $\mathbb{N}$ computable from a function $f \in \mathcal{C}[0,1]$ of non-total degree is a Scott set. In Section 9, we show that this is actually a characterization of the Scott sets. We finish with two examples of classical constructions adapted to prove results about continuous degrees. Let $\mathcal{S}$ be any Scott set. There are continuum many pairwise incomparable continuous degrees such that $\mathcal{S}$ is the Scott set induced by each one of them, i.e., $\mathcal{S}$ is the collection of subsets of $\mathbb{N}$ computable from each. Finally, for any $f \in[0,1]^{\mathbb{N}}$ of non-total degree, there is a $g \in[0,1]^{\mathbb{N}}$ such that $f$ and $g$ are incomparable and they compute exactly the same subsets of $\mathbb{N}$. This last theorem is proved using a variation of forcing with $\Pi_{1}^{0}$ classes.

$\S 2$. Computable analysis. We begin with a short introduction to those concepts from computable analysis which are necessary below. For a more complete introduction, see Weihrauch [43] or Pour-El and Richards [28]. Computable analysis was introduced almost simultaneously by Lacombe $[16,17]$ and Grzegorczyk $[9,10]$. Basic computable analysis provides definitions of computability for subsets of and functions on the real numbers. These notions can be generalized; we require computable structures on several spaces, so it is convenient to work with the espaces métriques récursifs (computable metric spaces) introduced by Lacombe [18]. A computable metric space is a complete metric space $\mathcal{M}$ together with a computable dense sequence $\mathcal{Q}^{\mathcal{M}}=\left\{q_{n}^{\mathcal{M}}\right\}_{n \in \mathbb{N}} \subseteq \mathcal{M}$ on which the metric is computable. ${ }^{3}$ In other words, there is a computable function $f: \mathbb{N}^{2} \times \mathbb{Q}^{+} \rightarrow \mathbb{Q}$ such that $\left|f(i, j, \varepsilon)-d_{\mathcal{M}}\left(q_{i}^{\mathcal{M}}, q_{j}^{\mathcal{M}}\right)\right|<\varepsilon$ for all $i, j \in \mathbb{N}$ and $\varepsilon \in \mathbb{Q}^{+}$. Recall that a metric space is separable if it has a countable dense subset and that a Polish space is a complete separable metric space, so our computable metric spaces are necessarily Polish spaces.

Paralleling Definition 1.1, a representation of $a \in \mathcal{M}$ is a function $\lambda: \mathbb{Q}^{+} \rightarrow \mathbb{N}$ such that, for all $\varepsilon \in \mathbb{Q}^{+}, d_{\mathcal{M}}\left(a, q_{\lambda(\varepsilon)}^{\mathcal{M}}\right)<\varepsilon$. This is equivalent to the standard Cauchy representation from [43]. Once an effective enumeration of $\mathbb{Q}^{+}$ has been fixed, representations can be viewed as elements of $\mathbb{N}^{\mathbb{N}}$, so classical computability can be applied. An element of $\mathcal{M}$ is called computable if it has a computable representation. If $\mathcal{M}_{0}$ and $\mathcal{M}_{1}$ are computable metric spaces, then a computable function from $\mathcal{M}_{0}$ to $\mathcal{M}_{1}$ is an effective map of representations of elements of $\mathcal{M}_{0}$ to representations of elements in $\mathcal{M}_{1}$, preserving

\footnotetext{
${ }^{3}$ Several variants on computable metric spaces appear in the literature. In particular, Weihrauch does not require $\mathcal{M}$ to be complete [43] — which means that it is not determined by the computable structure - and in an early treatment [42] he only requires the distance function on $\mathcal{Q}^{\mathcal{M}}$ to be right computable (i.e., the limit of a computable decreasing sequence).
} 
equivalence. More formally, $f: \mathcal{M}_{0} \rightarrow \mathcal{M}_{1}$ is a computable function if there is an index $e \in \mathbb{N}$ such that if $\lambda: \mathbb{Q}^{+} \rightarrow \mathbb{N}$ is a representation for $a \in \mathcal{M}_{0}$, then $\varphi_{e}^{\lambda}: \mathbb{Q}^{+} \rightarrow \mathbb{N}$ is a representation for $f(a) \in \mathcal{M}_{1}$, where as usual, $\varphi_{e}$ is the $e^{\text {th }}$ partial computable function (with oracle). Note that if $f$ is computable, then approximations to $f(a)$ are determined by suitable approximations to $a$; in other words, computable functions are continuous. Similarly, $\psi: \mathcal{M}_{0} \rightarrow \mathcal{M}_{1}$ is a partial computable function if there is an index $e \in \mathbb{N}$ such that if $\lambda$ is a representation for $a \in \mathcal{M}_{0}$, then either $\psi(a) \downarrow$ and $\varphi_{e}^{\lambda}$ is a representation for $\psi(a) \in \mathcal{M}_{1}$, or $\psi(a) \uparrow$ and $\varphi_{e}^{\lambda}$ is not total. We say that $\varphi_{e}$ induces $\psi$. As with total computable functions, partial computable functions are continuous on their domains. Finally, given computable metric spaces $\mathcal{M}_{0}$ and $\mathcal{M}_{1}$, there is a natural computable structure on the product $\mathcal{M}_{0} \times \mathcal{M}_{1}=\left\{a \oplus b \mid a \in \mathcal{M}_{0}\right.$ and $\left.b \in \mathcal{M}_{1}\right\}$. Simply take $d_{\mathcal{M}_{0} \times \mathcal{M}_{1}}\left(a_{0} \oplus a_{1}, b_{0} \oplus b_{1}\right)=\max \left\{d_{\mathcal{M}_{0}}\left(a_{0}, b_{0}\right), d_{\mathcal{M}_{1}}\left(a_{1}, b_{1}\right)\right\}$ and let $q_{\langle i, j\rangle}^{\mathcal{M}_{0} \times \mathcal{M}_{1}}=q_{i}^{\mathcal{M}_{0}} \oplus q_{j}^{\mathcal{M}_{1}}$.

EXAMPLES. We describe the computable metric spaces which are most important to us. We do not give explicit enumerations of $\mathcal{Q}^{\mathcal{M}}$ in these examples; any reasonable enumerations will suffice.

(a) $2^{\mathbb{N}}$ (subsets of $\mathbb{N}$ ) under the prefix metric:

$$
d(A, B)= \begin{cases}2^{-n}, & \text { if } n=(\mu n)[A(n) \neq B(n)] \\ 0, & \text { if } A=B\end{cases}
$$

Take $\mathcal{Q}^{2^{\mathbb{N}}}$ to be the finite sets. This is the Cantor space. Similarly, $\mathbb{N}^{\mathbb{N}}$ under the prefix metric gives us the Baire space. Define $\mathcal{Q}^{\mathbb{N}^{\mathbb{N}}}$ to be the set of functions with finite support.

These are the domains of classical computability theory with their standard computable structure. In particular, the computable elements of $2^{\mathbb{N}}$ and $\mathbb{N}^{\mathbb{N}}$ are exactly the computable sets and functions, and a partial function $\psi: \mathbb{N}^{\mathbb{N}} \rightarrow \mathbb{N}^{\mathbb{N}}$ is computable iff there is an index $e \in \mathbb{N}$ such that $(\forall f)\left[\psi(f) \downarrow=\varphi_{e}^{f} \Longleftrightarrow \varphi_{e}^{f}\right.$ is total].

(b) $\mathbb{R}$ with the standard metric and $\mathcal{Q}^{\mathbb{R}}=\mathbb{Q}$. Naturally, we can extend this space to $\mathbb{R}^{n}$ or restrict it to $[0,1]$.

This provides definitions of computability for reals and for functions on $\mathbb{R}$ which agree with the standard notions from computable analysis.

(c) $\mathcal{C}[0,1]$ (the continuous functions on $[0,1]$ ) under the uniform metric:

$$
d(f, g)=\max _{x \in[0,1]}|f(x)-g(x)| .
$$

We take $\mathcal{Q}^{\mathcal{C}[0,1]}$ to be the polygonal functions having segments with rational endpoints. Alternately, we could take $\mathcal{Q}^{\mathcal{C}[0,1]}$ to be the rational polynomials on $[0,1]$, which gives the same computable structure on $\mathcal{C}[0,1]$ (see Caldwell and Pour-El for details [26]). 
The computable elements of $\mathcal{C}[0,1]$ are exactly the total computable functions $[0,1] \rightarrow \mathbb{R}$.

(d) $[0,1]^{\mathbb{N}}$ (sequences of reals from $\left.[0,1]\right)$ under the metric

$$
d(\alpha, \beta)=\sum_{n \in \mathbb{N}}|\alpha(n)-\beta(n)| / 2^{n} .
$$

This metric induces the product topology on $[0,1]^{\mathbb{N}}$, producing a compact space known as the Hilbert cube. Let $\mathcal{Q}^{[0,1]^{\mathbb{N}}}$ be the finitely non-zero sequences of rationals from $[0,1]$.

The importance of $[0,1]^{\mathbb{N}}$ to the study of the degrees of continuous functions will become clear. Although $\mathcal{C}[0,1]$ is the space in which we are primarily interested, $[0,1]^{\mathbb{N}}$ is the space with which we primarily work.

$\S 3$. The continuous degrees. We are now ready to define the degrees of continuous functions, and in fact, of arbitrary members of computable metric spaces. It is convenient to define an inclusive notion of relative computability so that we may compare subsets of $\mathbb{N}$, real numbers, continuous functions on $[0,1]$, and infinite sequences of reals.

Definition 3.1. Given $a \in \mathcal{M}_{0}$ and $b \in \mathcal{M}_{1}, a \leq_{r} b$ ( $a$ is representation reducible to $b)$ if there is an index $e \in \mathbb{N}$ such that $\varphi_{e}^{\lambda}$ is a representation of $a$ for every representation $\lambda$ of $b$. If $a \leq_{r} b$ and $b \leq_{r} a$, then $a \equiv_{r} b$ ( $a$ and $b$ are representation equivalent).

It is clear that $\leq_{r}$ is reflexive and transitive. Corollary 4.3 will permit us to drop the uniformity from the definition. In other words, it states that $a \leq_{r} b$ iff every representation of $b$ computes a representation of $a$. Another equivalent formulation follows from the following result. Recall from the previous section that $\varphi_{e}$ is said to induce a partial computable function $\psi: \mathcal{M}_{0} \rightarrow \mathcal{M}_{1}$ if whenever $\lambda$ is a representation of $a \in \mathcal{M}_{0}$, then either $\psi(a) \downarrow$ and $\varphi_{e}^{\lambda}$ represents $\psi(a) \in \mathcal{M}_{1}$, or $\psi(a) \uparrow$ and $\varphi_{e}^{\lambda}$ is not total.

Proposition 3.2. Let $\mathcal{M}_{0}$ and $\mathcal{M}_{1}$ be computable metric spaces. There is a computable $\tau: \mathbb{N} \rightarrow \mathbb{N}$ such that, for all $e \in \mathbb{N}$ :

(a) $\varphi_{\tau(e)}$ induces a partial computable function $\psi_{e}: \mathcal{M}_{0} \rightarrow \mathcal{M}_{1}$, and

(b) if for every representation $\lambda$ of $a \in \mathcal{M}_{0}, \varphi_{e}^{\lambda}$ is a representation of $b \in \mathcal{M}_{1}$, then $\psi_{e}(a)=b$.

Moreover, $\tau$ is uniform in the computable presentations of $\mathcal{M}_{0}$ and $\mathcal{M}_{1}$.

This implies that $a \leq_{r} b$ iff there is a partial computable $\psi: \mathcal{M}_{1} \rightarrow \mathcal{M}_{0}$ such that $\psi(b)=a$. We omit the proof of Proposition 3.2. It is somewhat tedious and the result plays no role below.

Definition 3.3. The continuous degrees are the equivalence classes under $\equiv_{r}$ of elements of computable metric spaces. We use $\leq$ for the induced partial order. 
The continuous degree of $a \in \mathcal{M}$ is written as $\operatorname{deg}_{r}(a)$. If $a \in \mathcal{M}_{0}$ and $b \in \mathcal{M}_{1}$, then define $\operatorname{deg}_{r}(a) \cup \operatorname{deg}_{r}(b)=\operatorname{deg}_{r}(a \oplus b)$. It should be clear that if $a \equiv_{r} c$ and $b \equiv_{r} d$, then $(a \oplus b) \equiv_{r}(c \oplus d)$; so $\operatorname{deg}_{r}(a) \cup \operatorname{deg}_{r}(b)$ is well defined. Note also that $\operatorname{deg}_{r}(a) \cup \operatorname{deg}_{r}(b)$ is the least upper bound of $\operatorname{deg}_{r}(a)$ and $\operatorname{deg}_{r}(b)$.

We say that a continuous degree is total if it contains a subset of $\mathbb{N}$. Restricted to elements of $2^{\mathbb{N}}$ and $\mathbb{N}^{\mathbb{N}}$, representation reducibility coincides with Turing reducibility. Therefore, the total degrees are an embedded copy of the Turing degrees in the continuous degrees.

Note that representation reducibility applies to representations, which can themselves be viewed as elements of $\mathbb{N}^{\mathbb{N}}$ (once we have chosen an effective enumeration of $\mathbb{Q}^{+}$). In particular, this allows us to compare an element of a computable metric space to its own representations. The following proposition, which is easily verified, tells us that representations behave very naturally under representation reducibility.

Proposition 3.4. Let $m$ be an element of a computable metric space $\mathcal{M}$.

(a) If $\lambda$ is a representation of $m$, then $m \leq_{r} \lambda$.

(b) If $\mathbf{d e g}_{r}(m) \leq \mathbf{a}$ and $\mathbf{a}$ is total, then $m$ has a representation of degree $\leq \mathbf{a}$.

(c) The degree of $m$ is total iff $m$ has a representation $\lambda \equiv_{r} m$.

We show in Section 6 that there is a non-total continuous degree; in other words, not every continuous degree contains a subset of $\mathbb{N}$. Compare this to the following propositions, which prove that every continuous degree is populated by elements of $[0,1]^{\mathbb{N}}$ and, as the name suggests, of $\mathcal{C}[0,1]$ (indeed, real analytic elements of $\mathcal{C}[0,1])$.

Proposition 3.5. Every continuous degree contains an element of $[0,1]^{\mathbb{N}}$.

Proof. Let $\mathcal{M}$ be an arbitrary computable metric space. Define a function $f: \mathcal{M} \rightarrow[0,1]^{\mathbb{N}}$ by $f(m)=\bigoplus_{n} \min \left\{d\left(m, q_{n}^{\mathcal{M}}\right), 1\right\}$. Clearly $f$ is computable, so $f(m) \leq_{r} m$, for every $m \in \mathcal{M}$.

We must also prove that $m \leq_{r} f(m)$. Let $\lambda: \mathbb{Q}^{+} \rightarrow \mathbb{N}$ be a representation of $f(m)$. We construct a representation $\sigma: \mathbb{Q}^{+} \rightarrow \mathbb{N}$ for $m$. Take $\varepsilon \in \mathbb{Q}^{+}$ and let $\varepsilon_{0}=\min \{\varepsilon, 1\}$. Search for an $i \in \mathbb{N}$ such that $\lambda\left(\varepsilon_{0} / 2^{i+2}\right)(i)<\varepsilon_{0} / 2$. Assume such an $i$ exists. The fact that $\left|\lambda\left(\varepsilon_{0} / 2^{i+2}\right)-f(m)\right|<\varepsilon_{0} / 2^{i+2}$ implies that $\left|\lambda\left(\varepsilon_{0} / 2^{i+2}\right)(i)-f(m)(i)\right|<2^{i} \varepsilon_{0} / 2^{i+2}=\varepsilon_{0} / 4$, so $f(m)(i)<3 \varepsilon_{0} / 4<\varepsilon_{0} \leq 1$. Therefore, $d\left(m, q_{i}^{\mathcal{M}}\right)=f(m)(i)<\varepsilon_{0} \leq \varepsilon$, so we can let $\sigma(\varepsilon)=i$.

It remains to prove that the search for an appropriate $i$ succeeds. Because $\mathcal{Q}^{\mathcal{M}}$ is dense in $\mathcal{M}$, there is an $i \in \mathbb{N}$ such that $d\left(m, q_{i}^{\mathcal{M}}\right)<\varepsilon_{0} / 4$. But $f(m)(i)<\varepsilon_{0} / 4$ and $\left|\lambda\left(\varepsilon_{0} / 2^{i+2}\right)(i)-f(m)(i)\right|<\varepsilon_{0} / 4$ imply that $\lambda\left(\varepsilon_{0} / 2^{i+2}\right)(i)<\varepsilon_{0} / 2$. Therefore, for every $\varepsilon \in \mathbb{Q}^{+}, \sigma(\varepsilon) \downarrow$. So, $\sigma$ is a representation of $m$.

We have computed $\sigma$ uniformly from $\lambda$. This proves that $m \leq_{r} f(m)$. Therefore, $f(m) \in[0,1]^{\mathbb{N}}$ has the same degree as $m$, for all $m \in \mathcal{M}$. But $\mathcal{M}$ is arbitrary, so every continuous degree contains an element of $[0,1]^{\mathbb{N}}$.

Proposition 3.6. Every continuous degree contains an element of $\mathcal{C}[0,1]$. 
Proof. Let $\mathbf{v}$ be an arbitrary continuous degree. By the previous proposition, $\mathbf{v}$ contains a sequence $\alpha \in[0,1]^{\mathbb{N}}$. Define a function $f \in \mathcal{C}[0,1]$ such that $f\left(2^{-n}\right)=\alpha(n) / 2^{-n}$, for every $n$. Define $f$ be linear on every segment of the form $\left[2^{-n-1}, 2^{-n}\right]$ and, because continuity leaves us no choice, let $f(0)=0$. Then it is clear that $\alpha \equiv_{r} f$, so $f \in \mathbf{v}$.

A priori, we might expect that an understanding of the continuous degrees would require consideration of "pathological" continuous functions, but already we can see that this is not necessary. The function constructed in the proof above has a computable modulus of uniformity - in fact it is Lipschitz. Furthermore, it would not be hard, using the standard "smoothing" technique, to modify this construction to show that every continuous degree contains an infinitely differentiable $f \in \mathcal{C}[0,1]$ which computes the sequence of its own derivatives (i.e., $f^{(n)} \leq_{r} f$, uniformly in $n$ ), but we can do better with an even simpler construction.

Proposition 3.7. Every continuous degree contains a real-analytic function.

Proof. Let $\mathbf{v}$ be an arbitrary continuous degree and let $\alpha \in[0,1]^{\mathbb{N}}$ be a sequence of degree $\mathbf{v}$. Define a real-analytic function $f:[0,1] \rightarrow \mathbb{R}$ by $f(x)=$ $\sum_{n=0}^{\infty} \alpha(n) x^{n} / n$ !. It is clear that $f \leq_{r} \alpha$. Note that $\alpha(n)=f^{(n)}(0)$ for each $n$. But any real-analytic function computes the sequence of its own derivatives [27], so $\alpha \leq_{r} f$. Therefore, $\mathbf{v}$ contains a real-analytic element of $\mathcal{C}[0,1]$.

$\S 4$. The enumeration degrees. Kleene introduced a notion of computable reducibility for partial functions which coincides with Turing reduction on the total functions [14]. The degrees induced by this reducibility are called the partial degrees. Modifying Myhill's definition of the partial degrees [22], Rogers gave an alternate definition of the same degree structure as the enumeration degrees - the degrees of relative enumerability [30]. We follow Rogers.

Recall that $W_{e}$ is the $e^{\text {th }}$ computably enumerable set and $D_{e}$ is the finite set with canonical index $e$. We say that $A \subseteq \mathbb{N}$ is enumeration reducible to $B \subseteq \mathbb{N}$ $\left(A \leq_{e} B\right)$ if there exists a $z \in \mathbb{N}$ such that

$$
(\forall x)\left[x \in A \Longleftrightarrow(\exists u)\left[\langle x, u\rangle \in W_{z} \wedge D_{u} \subset B\right]\right]
$$

Informally, $A \leq_{e} B$ if there is effective procedure which, when given an enumeration of $B$-in any order - produces an enumeration of $A$. Write $A \equiv_{e} B$ if $A \leq_{e} B$ and $A \geq_{e} B$ and define the enumeration degrees to be the equivalence classes under $A \equiv_{e} B$.

If we identify partial functions with their graphs, enumeration reducibility induces the relation defined by Kleene. An enumeration degree is called total if it contains the graph of a total function, or equivalently, if it contains the graph of a characteristic function $\chi_{A}$, for some $A \subseteq \mathbb{N}$. It is well known that $A \leq_{T} B$ iff $\chi_{A} \leq_{e} \chi_{B}$. Therefore, the Turing degrees embed into the enumeration degrees as the total degrees. We can also embed the continuous degrees into the 


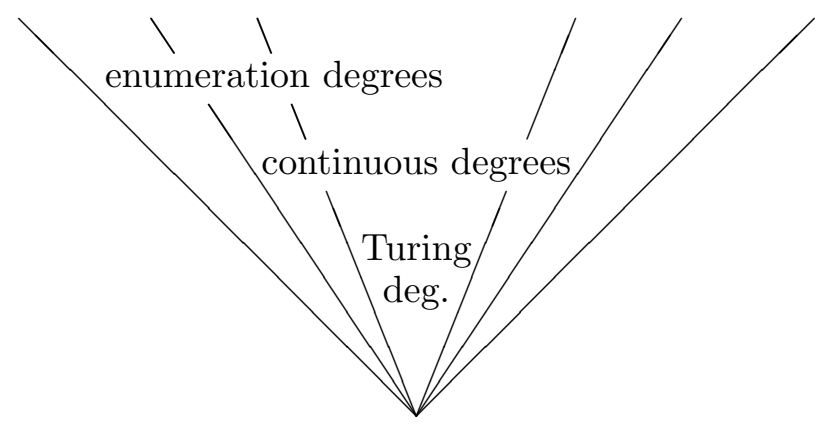

Figure 1. Nested degree structures.

enumeration degrees. If $\alpha \in[0,1]^{\mathbb{N}}$, define

$$
\Xi(\alpha)=\left\{\langle 0, i, j\rangle \mid q_{j}^{[0,1]}<\alpha(i)\right\} \cup\left\{\langle 1, i, j\rangle \mid q_{j}^{[0,1]}>\alpha(i)\right\} .
$$

Note that from a representation of a sequence $\alpha \in[0,1]^{\mathbb{N}}$ we can enumerate $\Xi(\alpha) \subseteq \mathbb{N}$ and from an enumeration of $\Xi(\alpha)$ we can compute a representation of $\alpha$. Furthermore, both of these computations can be done uniformly. These observations prove the following.

Proposition 4.1. If $\alpha, \beta \in[0,1]^{\mathbb{N}}$, then $\alpha \leq_{r} \beta$ iff $\Xi(\alpha) \leq_{e} \Xi(\beta)$.

Therefore, the continuous degrees form a natural substructure of the enumeration degrees. It is not hard to see that this embedding of the continuous degrees into the enumeration degrees agrees with the usual embedding of the Turing degrees (hence the total degrees are defined unambiguously). Furthermore, each of these embeddings preserves joins. From now on, we identify the continuous degrees with their image in the enumeration degrees and the Turing degrees with the total degrees. ${ }^{4}$ We prove below that both embeddings are nontrivial, as is implied by Figure 1 .

A useful equivalent formulation of enumeration reducibility is implicit in work of Selman [35]. This characterization was rediscovered by Rozinas [31].

Theorem 4.2. Let $A, B \subseteq \mathbb{N}$.

(a) (Selman, 1971) $A \leq_{e} B$ iff $(\forall C)[B$ is c.e. in $C \Longrightarrow A$ is c.e. in $C]$.

(b) (Rozinas, 1978) $A \leq_{e} B$ iff $(\forall C)\left[B \leq_{e} \chi_{C} \Longrightarrow A \leq_{e} \chi_{C}\right]$.

Note that $B$ is c.e. in $C$ iff $B \leq_{e} \chi_{C}$, so $(a)$ and $(b)$ are identical. These results essentially remove uniformity from the definition of enumeration reducibility ${ }^{5}$; we now apply this to representation reducibility.

\footnotetext{
${ }^{4}$ In particular, for $A \subseteq \mathbb{N}$ we consider $\operatorname{deg}_{T}(A)=\operatorname{deg}_{r}(A)=\operatorname{deg}_{e}\left(\chi_{A}\right)$, even though formally these are different sets.

${ }^{5}$ We can formalize this observation as follows. Rogers embeds the enumeration degrees into the Medvedev lattice using $\iota(A)=\left\{f \in \mathbb{N}^{\mathbb{N}} \mid\right.$ range $\left.(f)=A\right\}$ for $A \neq \emptyset[30]$. As a consequence of Theorem 4.2, Medvedev reducibility (which is uniform) [20] and Mučnik reducibility (which is non-uniform) [21] agree on the image of $\iota$.
} 
Corollary 4.3. Given $a \in \mathcal{M}_{0}$ and $b \in \mathcal{M}_{1}$, where $\mathcal{M}_{0}$ and $\mathcal{M}_{1}$ are computable metric spaces, $a \leq_{r} b$ iff every representation of $b$ computes a representation of $a$.

PRoOF. If $a \leq_{r} b$, then it is immediate that every representation of $b$ computes a representation of $a$. For the other direction, assume that $a \not_{r} b$. Choose $\alpha, \beta \in[0,1]^{\mathbb{N}}$ such that $\alpha \equiv_{r} a$ and $\beta \equiv_{r} b$. So $\alpha \not_{r} \beta$, which implies that $\Xi(\alpha) \leq_{e} \Xi(\beta)$. By Theorem $4.2(b)$, there is a $C \subseteq \mathbb{N}$ such that $\Xi(\beta) \leq_{e} \chi_{C}$ but $\Xi(\alpha) \not_{e} \chi_{C}$. Translating back, we get that $\beta \leq_{r} C$ and $\alpha \not_{r} C$. Unwinding further, $b \leq_{r} C$ and $a \leq_{r} C$. By Proposition 3.4, there is a representation $\lambda \leq_{r} C$ for $b$. But $a \leq_{r} \lambda$, so $\lambda$ does not compute any representation of $a$. $\dashv$

Next we return to the question motivating this investigation.

COROLlary 4.4. If $f \in \mathcal{C}[0,1]$ has non-total degree, then $f$ has no representation of least Turing degree.

Proof. Assume that $f$ has a representation $\lambda: \mathbb{Q}^{+} \rightarrow \mathbb{N}$ with least Turing degree among all representations of $f$. We know that $\lambda$ has total degree, so by Proposition 3.4(c), $\lambda$ has a representation $\sigma: \mathbb{Q}^{+} \rightarrow \mathbb{N}$ such that $\lambda \equiv_{r} \sigma$. Every representation of $f$ computes $\lambda$, hence computes $\sigma$. Therefore by Corollary 4.3, $\lambda \leq_{r} f$. But $f \leq_{r} \lambda$ by Proposition 3.4(a). So $f \equiv_{r} \lambda$, which means that $f$ has total degree.

We construct a non-total continuous degree in Section 6, answering Pour-El and Lempp's question in the negative. We finish this section with one more consequence of Theorem 4.2.

COROllary 4.5. Enumeration degrees (hence continuous degrees) are determined by the total degrees above them.

As it turns out, continuous degrees are not determined by the total degrees below them (see Theorem 9.5).

$\S 5$. Computably diagonalizable sequences. Having completed the preliminaries, the next two sections are devoted to our first main result: not every continuous degree is total. The problem is reduced to the construction of a sequence $\alpha \in[0,1]^{\mathbb{N}}$ with the unusual property that it cannot be diagonalized by a computable operator.

A sequence $\alpha \in[0,1]^{\mathbb{N}}$ is computably diagonalizable if there is an $x \in[0,1]$ such that $x \leq_{r} \alpha$ and $x \notin \operatorname{range}(\alpha)$. Put another way, if a sequence $\alpha \in[0,1]^{\mathbb{N}}$ is not computably diagonalizable, then $\alpha$ is a list of all reals in $[0,1]$ which are computable from itself. These sequences play an important role in the theory of the continuous degrees; by Proposition 5.3, a continuous degree is non-total iff it contains a sequence which is not computably diagonalizable. Thus, the existence of such a sequence is sufficient to answer Question 1.2. We construct a sequence which is not computably diagonalizable in the next section. 
The following lemma proves that we can compute a non-member of a sequence $\alpha \in[0,1]^{\mathbb{N}}$ from any representation of $\alpha$. Note that for a sequence to fail to be computably diagonalizable, it must be impossible to compute the same $x \notin$ range $(\alpha)$ from all representations (here we are using Corollary 4.3).

LEMMA 5.1. Let $\alpha \in[0,1]^{\mathbb{N}}$. If $\lambda$ is any representation of $\alpha$, then there is an $x \in[0,1]$ such that $x \leq_{r} \lambda$ (which is equivalent to $x \leq_{T} \lambda$ ) and $x \notin \operatorname{range}(\alpha)$.

Proof. Let $\lambda: \mathbb{Q}^{+} \rightarrow \mathbb{N}$ be any representation of $\alpha$. We construct a nested sequence of closed intervals $\left\{I_{n}\right\}_{n \in \mathbb{N}}$ computably in $\lambda$ such that, for each $n$, $\left|I_{n}\right|=3^{-n}$ and if $i<n$, then $\alpha(i) \notin I_{n}$. Let $I_{0}=[0,1]$. Assume that $I_{n}=[l, r]$ has been defined; we define $I_{n+1}$ by

$$
I_{n+1}= \begin{cases}{[l,(2 l+r) / 3],} & \text { if } \lambda\left(2^{-n} 3^{-n-2}\right)(n) \geq(l+r) / 2 \\ {[(l+2 r) / 3, r],} & \text { otherwise. }\end{cases}
$$

So $I_{n+1}$ is either the right third or left third of $I_{n}$. Also $\left|\lambda\left(2^{-n} 3^{-n-2}\right)(n)-\alpha(n)\right|<$ $3^{-n-2}$, so $\alpha(n) \notin I_{n+1}$. Let $x=\bigcap I_{n}$. Then $x \notin \operatorname{range}(\alpha)$ and $x$ is computable from $\lambda$, as required.

Next we give a simple condition under which a sequence has total degree.

LEMmA 5.2. If $\alpha \in[0,1]^{\mathbb{N}}$ contains no binary rationals, then it has total degree.

Proof. We can uniformly compute the (unique) binary expansion of each $\alpha(n)$ from any representation of $\alpha$. Therefore, if we define a set $A \subseteq \mathbb{N}$ by

$$
A=\left\{\langle n, k\rangle \mid \text { the } k^{\text {th }} \text { digit in the binary expansion of } \alpha(n) \text { is } 1\right\},
$$

then $A \equiv_{r} \alpha$.

Proposition 5.3. A continuous degree is non-total iff it contains a sequence $\alpha \in[0,1]^{\mathbb{N}}$ which is not computably diagonalizable.

Proof. First assume that $\alpha \in[0,1]^{\mathbb{N}}$ has total degree. By Proposition 3.4(c), $\alpha$ has a representation $\lambda \equiv_{r} \alpha$. By Lemma 5.1, there is an $x \in[0,1]$ with $x \leq_{r} \lambda$ and $x \notin \operatorname{range}(\alpha)$. So $x \leq_{r} \alpha$, which means that $\alpha$ is computably diagonalizable.

Next, assume that $\mathbf{v}$ is a continuous degree containing only computably diagonalizable sequences. By Proposition 3.5, we can take $\alpha \in[0,1]^{\mathbb{N}}$ such that $\operatorname{deg}_{r}(\alpha)=\mathbf{v}$. Let $\left\{b_{i}\right\}_{i \in \mathbb{N}}$ be an effective enumeration of the nonnegative binary rationals. Define $\beta \in[0,1]^{\mathbb{N}}$ by $\beta=\bigoplus_{\langle i, j, k\rangle} \min \left\{\alpha(i) b_{j}+b_{k}, 1\right\}$. Clearly $\alpha \equiv_{r} \beta$, so $\beta \in \mathbf{v}$. By assumption, every sequence of degree $\mathbf{v}$ is computably diagonalizable, hence there is an $r \in[0,1]$ such that $r \leq_{r} \beta$ and $r \notin \operatorname{range}(\beta)$.

We claim that $r /(\beta(n)+1)$ cannot be a binary rational, for any $n \in \mathbb{N}$. Assume, for a contradiction, that $r /(\beta(n)+1)=b_{m}$. There are two cases; either $r=(\beta(n)+1) b_{m}=\left(\left(\alpha(i) b_{j}+b_{k}\right)+1\right) b_{m}=\alpha(i)\left(b_{j} b_{m}\right)+\left(b_{k} b_{m}+b_{m}\right)$, where $n=\langle i, j, k\rangle$, or $r=(\beta(n)+1) b_{m}=2 b_{m}$. In both cases, $r$ is of the form $\alpha(i) c_{1}+c_{2}$, where $c_{1}$ and $c_{2}$ are nonnegative binary rationals. Therefore, 
$r \in \operatorname{range}(\beta)$, which is a contradiction. This implies that $r /(\beta(n)+1) \in[0,1]$ is never a binary rational.

Therefore, the sequence $\gamma \in[0,1]^{\mathbb{N}}, \gamma=\bigoplus_{n} r /(\beta(n)+1)$ contains no binary rationals. By Lemma 5.2, $\operatorname{deg}_{r}(\gamma)$ is total. But clearly $\gamma \equiv_{r} \beta$, so $\mathbf{v}$ is total. $\dashv$

§6. A non-total continuous degree. In this section, we construct a sequence of reals which is not computably diagonalizable. We require the following classical fixed point theorem for multivalued functions on $[0,1]^{\mathbb{N}}$. Recall that a subset $\mathcal{S} \subseteq[0,1]^{\mathbb{N}}$ is convex if whenever $x \in[0,1]$ and $\alpha, \beta \in \mathcal{S}$, then $x \alpha+(1-x) \beta \in \mathcal{S}$ (where the sum is defined pointwise).

THEOREM 6.1. Assume that $\Psi:[0,1]^{\mathbb{N}} \rightarrow[0,1]^{\mathbb{N}}$ is a multivalued function with a closed graph such that $\Psi(\alpha)$ is nonempty and convex for each $\alpha \in[0,1]^{\mathbb{N}}$. Then there is a fixed point $\alpha$ of $\Psi$ (i.e., $\alpha \in \Psi(\alpha))$.

The generalization of Brouwer's fixed point theorem to compact convex regions in Banach spaces was done by Schauder [33], though already in 1922, Birkhoff and Kellogg had proved important special cases [3]. Kakutani generalized Brouwer's theorem to multivalued functions with closed graphs which take points to nonempty convex sets [13]. The theorems of Schauder and Kakutani were unified by Bohnenblust and Karlin [4], but this is not the earliest result which implies Theorem 6.1. That honor likely goes to a purely topological generalization of Kakutani's fixed point theorem proved by Eilenberg and Montgomery [8].

The following lemma provides the function to which Theorem 6.1 is applied.

LEMMA 6.2. There is a multivalued function $\Psi:[0,1]^{\mathbb{N}} \rightarrow[0,1]^{\mathbb{N}}$ with a closed graph and nonempty, convex images such that, for all $e \in \mathbb{N}, \alpha \in[0,1]^{\mathbb{N}}$ and $\beta \in \Psi(\alpha)$, if for every representation $\lambda$ of $\alpha, \varphi_{e}^{\lambda}$ is a representation of $x \in[0,1]$, then $\beta(e)=x$.

The intuition behind the construction of $\Psi$ is fairly simple. It must be shown that each $\varphi_{e}$ "induces" a multivalued function $\psi_{e}:[0,1]^{\mathbb{N}} \rightarrow[0,1]$ such that for each $\alpha \in[0,1]^{\mathbb{N}}, \psi_{e}(\alpha)$ is the smallest closed interval consistent with the behavior of $\varphi_{e}$ on (certain special) representations of $\alpha$. Then we simply let $\Psi=\bigoplus_{e} \psi_{e}$ to satisfy the lemma. We describe the details of this construction below, but first we combine the lemma with Theorem 6.1 to prove the main result of this section.

ThEOREM 6.3. There is a sequence $\alpha \in[0,1]^{\mathbb{N}}$ which is not computably diagonalizable. In particular, no fixed point of the multivalued function $\Psi:[0,1]^{\mathbb{N}} \rightarrow$ $[0,1]^{\mathbb{N}}$ constructed in Lemma 6.2 is computably diagonalizable.

Proof. By Theorem 6.1, $\Psi$ has a fixed point $\alpha \in[0,1]^{\mathbb{N}}$. We must prove that $\alpha$ is not computably diagonalizable. Recall that $\alpha \in[0,1]^{\mathbb{N}}$ is not computably diagonalizable if $x \in \operatorname{range}(\alpha)$ whenever $x \in[0,1]$ and $x \leq_{r} \alpha$. Assume that $x \in[0,1]$ and $x \leq_{r} \alpha$. So there is an $e \in \mathbb{N}$ such that if $\lambda$ is a representation 
of $\alpha$, then $\varphi_{e}^{\lambda}$ is a representation of $x$. From the lemma, using the fact that $\alpha \in \Psi(\alpha), \alpha(e)=x$. In particular, $x \in$ range $(\alpha)$. But $x$ was arbitrary, so $\alpha$ is not computably diagonalizable.

Not only is the $\alpha$ constructed above not computably diagonalizable, it satisfies an apparently much stronger property: if $x \in[0,1]$ and $\varphi_{e}$ witnesses the fact that $x \leq_{r} \alpha$ (i.e., $\varphi_{e}^{\lambda}(\alpha)$ is a representation of $x$ for every representation $\lambda$ of $\alpha$ ), then $\alpha(e)=x$. Such a sequence could be called diagonally not computably diagonalizable. We do not know if this stronger property plays an important theoretical role.

Together with Proposition 5.3 and Corollary 4.4, Theorem 6.3 yields two of the primary results of this paper, and in particular, the answer to Question 1.2.

COROllary 6.4. There is a non-total continuous degree.

COROLlary 6.5. There is a continuous function $f \in \mathcal{C}[0,1]$ which does not have a representation of least Turing degree.

We now turn to the construction of a multivalued function $\Psi:[0,1]^{\mathbb{N}} \rightarrow[0,1]^{\mathbb{N}}$ satisfying Lemma 6.2. The graph of $\Psi$ will not only be closed but, in an appropriate sense, will be a $\Pi_{1}^{0}$ class. Recall that a $\Pi_{1}^{0}$ class (of sets) is just an effectively closed subset of $2^{\mathbb{N}}$. More formally, a tree is a subset of $2^{<\mathbb{N}}$ which is closed downward under initial segments. If $T \subseteq 2^{<\mathbb{N}}$ is a tree, we write $[T] \subseteq 2^{\mathbb{N}}$ for the set of infinite paths through $T$ (which we identify with subsets of $\mathbb{N}$ ). Note that the closed subsets of $2^{\mathbb{N}}$ are exactly the sets of the form $[T]$, for trees $T \subseteq 2^{<\mathbb{N}}$. When $T$ is a computable tree, then we call $[T]$ a $\Pi_{1}^{0}$ class. Equivalently, the $\Pi_{1}^{0}$ classes are the $\Pi_{1}^{0}$-definable subsets of $2^{\mathbb{N}}$. More information on $\Pi_{1}^{0}$ classes can be found in $[5,6]$.

Let $\pi: 2^{\mathbb{N}} \rightarrow[0,1]$ be defined by $\pi(A)=\sum_{i \in A} 2^{-i-1}$, for $A \subseteq \mathbb{N}$. In other words, $A$ encodes a binary expansion of $\pi(A)$. Now define $\Pi: 2^{\mathbb{N}} \rightarrow[0,1]^{\mathbb{N}}$ by $\Pi(A)=\bigoplus_{n \in \mathbb{N}} \pi\left(A_{(n)}\right)$, where $A_{(n)}=\{i \mid\langle n, i\rangle \in A\}$. If $\Pi(A)=\alpha$, then we call $A$ a $b$-representation of $\alpha$. It is important to note that we can uniformly compute representations of sequences from b-representations. ${ }^{6}$

LEMmA 6.6. There is a computable $\Gamma: 2^{\mathbb{N}} \rightarrow \mathbb{N}^{\mathbb{Q}^{+}}$such that if $A \subseteq \mathbb{N}$ is a b-representation of $\alpha \in[0,1]^{\mathbb{N}}$, then $\Gamma(A)$ is a representation of $\alpha$.

Proof. Compute $\Gamma$ as follows. Let $A \subseteq \mathbb{N}$ and $\alpha=\Pi(A)$. For $\varepsilon \in \mathbb{Q}^{+}$, choose $n \in \mathbb{N}$ least such that $(n+2) 2^{-n}<\varepsilon$. Let $\beta=\bigoplus_{i \in \mathbb{N}} \sum_{k \in A_{(i)}, k \leq n-i} 2^{-k-1}$. For

\footnotetext{
${ }^{6}$ The reverse is not true, so b-representation is not equivalent - again in the sense of Kreitz and Weihrauch $[15,43]$ - to our standard representation for sequences. In fact, there is a computable $\alpha \in[0,1]^{\mathbb{N}}$ without a computable b-representation. We use b-representations because for every $\alpha \in[0,1]^{\mathbb{N}}$, the set $\Pi^{-1}(\alpha) \subseteq 2^{\mathbb{N}}$ of b-representations of $\alpha$ is compact. It is worth noting that there are representations equivalent to the standard one which also have this property.
} 
$i \leq n,|\alpha(i)-\beta(i)| \leq 2^{-n+i}$, so

$$
d_{[0,1]^{\mathbb{N}}}(\alpha, \beta)=\sum_{i=0}^{\infty}|\alpha(i)-\beta(i)| / 2^{i} \leq \sum_{i=0}^{n} 2^{-n+i} / 2^{i}+\sum_{i>n} 1 / 2^{i}=(n+2) 2^{-n}<\varepsilon .
$$

Note that $\beta=q_{m}^{[0,1]^{\mathbb{N}}}$ for some $m \in \mathbb{N}$. Define $\Gamma(A)(\varepsilon)=m$. Therefore, $\Gamma(A)$ is a representation of $\alpha$. Clearly, $\Gamma: 2^{\mathbb{N}} \rightarrow \mathbb{N}^{\mathbb{Q}^{+}}$is computable.

In the following proof, we construct $\Psi$ so that

$$
\{A \subseteq \mathbb{N} \mid A=B \oplus C \text { where } \Pi(C) \in \Psi(\Pi(B))\}
$$

is a $\Pi_{1}^{0}$ class. Although this more than the lemma requires, it will be very useful in Sections 8 and 9. It is worth noting that the definition of $\Pi_{1}^{0}$ class can be generalized from subsets of $2^{\mathbb{N}}$ to subsets of arbitrary computable metric spaces and even effective topological spaces [18], providing a natural notion of effectiveness for closed sets. These classes (which Weihrauch calls co-r.e. closed sets [43]) play an important role in computable analysis. The generalization would not significantly simplify our presentation, so we avoid it here. However, under the broader definition, the graph of $\Psi$ would itself be a $\Pi_{1}^{0}$ class.

Proof of Lemma 6.2. For $A \subseteq \mathbb{N}$ and $e \in \mathbb{N}$, we inductively define closed intervals $I(A ; e, n)$ as follows. Let $I(A ; e, 0)=[0,1]$. For $n>0$, let

$$
E=\left[\varphi_{e}^{\Gamma(A)}\left(2^{-n}\right)-2^{-n}, \varphi_{e}^{\Gamma(A)}\left(2^{-n}\right)+2^{-n}\right] .
$$

Note that $E$ may not be defined; if it is, then let

$$
I(A ; e, n)= \begin{cases}I(A ; e, n-1) \cap E, & \text { if } I(A ; e, n-1) \cap E \neq \emptyset \\ I(A ; e, n-1), & \text { otherwise. }\end{cases}
$$

Then $I$ is a partial computable function from $2^{\mathbb{N}} \times \mathbb{N}^{2}$ to rational subintervals of $[0,1]$. Define $I(A ; e, \omega)=\bigcap_{n \in \mathbb{N}} I(A ; e, n)$, where we ignore undefined terms and interpret the empty intersection as $[0,1]$. Then $I(A ; e, \omega)$ is a nested intersection of (possibly infinitely many) compact intervals, so it is either a closed interval or a single point. In particular, $I(A ; e, \omega)$ is not empty. Note that if $\varphi_{e}^{\Gamma(A)}$ is a representation of $x \in[0,1]$, then $I(A ; e, \omega)=\{x\}$. Informally, $I(A ; e, \omega)$ captures the ambiguity of $\varphi_{e}^{\Gamma(A)}$.

Even if $A, B \in \mathbb{N}$ are b-representations of the same sequence, $I(A ; e, \omega)$ and $I(A ; e, \omega)$ need not be equal. We next want to define closed intervals $J(A ; e, n, m)$ which address this irregularity. First define $\sigma, \tau \in 2^{<\mathbb{N}}$ to be compatible, written as compat $(\sigma, \tau)$, if they can be extended to b-representations of the same sequence. Compatibility is clearly decidable. Note that for $A \subseteq \mathbb{N}$, if $\sigma \in 2^{<\mathbb{N}}$ can not be extended to a b-representation of $\Pi(A)$, then for large enough $m \in \mathbb{N}$, $A \uparrow m$ and $\sigma$ are not compatible. Now define the convex union of closed intervals $[a, b]$ and $[c, d]$ to be $[a, b] \sqcup[c, d]=[\min \{a, c\}, \max \{b, d\}]$. For $A \subseteq \mathbb{N}$ and $e, n, m \in \mathbb{N}$, let

$$
J(A ; e, n, m)=\bigsqcup\left\{I(\sigma ; e, n) \mid \sigma \in\{0,1\}^{m} \text { and } \operatorname{compat}(\sigma, A \uparrow m)\right\},
$$


where $J(A ; e, n, m)$ is defined only if all the terms in the convex union are defined. It is clear from the definition that $J(A ; e, n, m)$ is a partial computable function. Observe that if $J(A ; e, n, m)$ is defined and $\widehat{m}>m$, then $J(A ; e, n, \widehat{m})$ is also defined and $J(A ; e, n, \widehat{m}) \subseteq J(A ; e, n, m)$; the convex union will not gain new (distinct) terms but it may lose terms corresponding to $\sigma \in 2^{m}$ which cannot be extended to a b-representation of $\Pi(A)$. Define $J(A ; e, n, \omega)=\bigcap_{m \in \mathbb{N}} J(A ; e, n, m)$, where the intersection ignores undefined terms but we interpret the empty intersection as undefined. We claim that

$$
J(A ; e, n, \omega)=\bigsqcup\{I(B ; e, n) \mid B \subseteq \mathbb{N} \text { and } \Pi(B)=\Pi(A)\} .
$$

This is a consequence of compactness. First assume that there is a $B \subseteq \mathbb{N}$ such that $\Pi(B)=\Pi(A)$ and $I(B ; e, n) \uparrow$. In this case, the right side of the equality is clearly undefined. Also note that $J(A ; e, n, m)$ is undefined for every $m$. Hence, $J(A ; e, n, \omega)$ is undefined, so the equality holds. Therefore, we may assume that for every b-representation $B \subseteq \mathbb{N}$ of $\Pi(A), I(B \uparrow k ; e, n) \downarrow$ for large enough $k \in \mathbb{N}$. But the class of b-representations of $\Pi(A)$ is a closed subset of $2^{\mathbb{N}}$. So by compactness, we can find a single $k$ which works for all $B$. Now choose a number $m \geq k$ large enough that every $\sigma \in 2^{k}$ which does not extend to a b-representation of $\Pi(A)$ is incompatible with $A \uparrow m$. For this $m, J(A ; e, n, m)=J(A ; e, n, \omega)=\bigsqcup\{I(B ; e, n) \mid B \subseteq \mathbb{N}$ and $\Pi(B)=\Pi(A)\}$, proving the claim. It follows that if $A, B \in \mathbb{N}$ are b-representations of the same sequence, then $J(A ; e, n, \omega)=J(B ; e, n, \omega)$ (or both are undefined).

Let $J(A ; e, \omega, \omega)=\bigcap_{n \in \mathbb{N}} J(A ; e, n, \omega)=\bigcap_{n, m \in \mathbb{N}} J(A ; e, n, m)$, where we ignore undefined terms and take the empty intersection to be $[0,1]$. So $J(A ; e, \omega, \omega) \subseteq$ $[0,1]$ is either a closed interval or a single point and $\Pi(A)=\Pi(B)$ implies that $J(A ; e, \omega, \omega)=J(B ; e, \omega, \omega){ }^{7}$

Fix $A \subseteq \mathbb{N}$ and $e \in \mathbb{N}$ and assume that for every representation $\lambda$ of $\Pi(A)=$ $\alpha \in[0,1]^{\mathbb{N}}, \varphi_{e}^{\lambda}$ represents $x \in[0,1]$. So a fortiori, $\varphi_{e}^{\Gamma(B)}$ represents $x \in[0,1]$ for every b-representation $B \subseteq \mathbb{N}$ of $\alpha$. We claim that $J(A ; e, \omega, \omega)=\{x\}$. Fix $n \in \mathbb{N}$. We know that for every b-representation $B \subseteq \mathbb{N}$ of $\alpha, I(B ; e, n)$ is defined, contains $x$ and has length at most $2^{-n+1}$. Therefore, $J(A ; e, n, \omega)$ contains $x$ and has length less than $2^{-n+2}$. It follows that $J(A ; e, \omega, \omega)=\{x\}$.

We can now define the multivalued function $\Psi:[0,1]^{\mathbb{N}} \rightarrow[0,1]^{\mathbb{N}}$ by $\Psi(\alpha)=$ $\bigoplus_{e \in \mathbb{N}} J(A ; e, \omega, \omega)$, where $A \subseteq \mathbb{N}$ is any b-representation of $\alpha$. Note that $\Psi(\alpha)$ does not depend on the choice of $A$. It is clear from the properties of $J(A ; e, \omega, \omega)$ that $\Psi$ has nonempty, convex images and that for all $e \in \mathbb{N}, \alpha \in[0,1]^{\mathbb{N}}$ and $\beta \in \Psi(\alpha)$, if $\varphi_{e}^{\lambda}$ is a representation of $x \in[0,1]$ for every representation $\lambda$ of $\alpha$, then $\beta(e)=x$. To complete the proof of the lemma we must show that the graph of $\Psi$ is closed.

\footnotetext{
${ }^{7}$ The informal description of this construction on page 12 mentions a multivalued function $\psi_{e}:[0,1]^{\mathbb{N}} \rightarrow[0,1]$ which is "induced" by $\varphi_{e}$. That function, in the notation of the construction, is given by $\psi_{e}(\alpha)=J(A ; e, \omega, \omega)$, where $A \subseteq \mathbb{N}$ is any b-representation of $\alpha$ (and the choice of $A$ is irrelevant).
} 
Define a class $\mathcal{G} \subseteq 2^{\mathbb{N}}$ by

$$
A \oplus B \in \mathcal{G} \Longleftrightarrow(\forall e, n, m)\left[J(A ; e, n, m) \downarrow \Longrightarrow \pi\left(B_{(e)}\right) \in J(A ; e, n, m)\right] .
$$

This is a $\Pi_{1}^{0}$ definition $\left(J(A ; e, n, m) \downarrow\right.$ is $\Sigma_{1}^{0}$ and $\pi\left(B_{(e)}\right) \in J(A ; e, n, m)$ is $\left.\Pi_{1}^{0}\right)$. Therefore, $\mathcal{G}$ is a $\Pi_{1}^{0}$ class. In particular, $\mathcal{G}$ is a closed (hence compact) subset of $2^{\mathbb{N}}$. But note that $\mathcal{G}=\{A \oplus B \subseteq \mathbb{N} \mid \Pi(B) \in \Psi(\Pi(A))\}$. In other words, the graph of $\Psi$ is the image of $\mathcal{G}$ under $\Pi \times \Pi: 2^{\mathbb{N}} \times 2^{\mathbb{N}} \rightarrow[0,1]^{\mathbb{N}} \times[0,1]^{\mathbb{N}}$. But the continuous image of a compact set is compact, so the graph of $\Psi$ is closed. $\dashv$

$\S 7$. Diagonalizing sequences of computable reals. In the introduction, we drew an analogy between continuous functions with non-total degree and countable structures with no Turing degree - in other words, with no presentation of least Turing degree - as studied by L. J. Richter [29]. We now make a closer comparison of these phenomena. Richter produced structures which have no Turing degree in two ways. For some theories (e.g., partial orders and abelian groups), she showed that there were structures realizing every possible enumeration degree. Because there are non-total enumeration degrees, such theories have structures with no Turing degree. For other theories (e.g., linear orders and boolean algebras), she showed that every structure has a minimal pair of presentations. For such theories, no structure can compute a non-computable subset of $\mathbb{N}$, so no non-computable structure has a Turing degree. In this section, we shown that neither approach can be used to produce a continuous function with non-total degree. It follows from Corollary 7.3 that not every enumeration degree is realized by a continuous function and that every non-computable continuous function computes a non-computable subset of $\mathbb{N}$.

We turn to the details.

Definition 7.1. A non-total enumeration degree is quasi-minimal if $\mathbf{0}$ is the only total degree below it.

Medvedev established the existence of quasi-minimal degrees, and thus of nontotal enumeration degrees [20]. As Myhill later showed, the subsets of $\mathbb{N}$ of quasi-minimal degree form a co-meager set [22]; quasi-minimal enumeration degrees are the rule, not the exception. Our goal is to prove that the continuous degrees are a proper substructure of the enumeration degrees by showing that no continuous degree is quasi-minimal. Again we exploit the notion of computable diagonalizability.

THEOREM 7.2. Every sequence $\alpha \in[0,1]^{\mathbb{N}}$ of computable reals is computably diagonalizable.

Before proving the theorem we consider its consequences.

Corollary 7.3. No continuous degree is quasi-minimal.

Proof. Assume that $\mathbf{v}$ is a quasi-minimal continuous degree. Since $\mathbf{v}$ is nontotal, by Proposition 5.3, v contains a sequence $\alpha \in[0,1]^{\mathbb{N}}$ which is not computably diagonalizable. For each coordinate $\alpha(i)$ of $\alpha$, we have that $\alpha(i) \leq_{r} \alpha$. 
But $\alpha(i) \in[0,1]$ has total degree, so it must be computable by the quasiminimality of $\mathbf{v}$. Therefore, $\alpha \in \mathbf{v}$ is a sequence of computable reals. By Theorem 7.2, $\alpha$ is computably diagonalizable, but this is a contradiction.

In other words, every non-computable $f \in \mathcal{C}[0,1]$ computes a non-computable subset of $\mathbb{N}$. As explained above, Corollary 7.3 implies that not every enumeration degree is continuous. Even better, we can distinguish the enumeration degrees and continuous degrees as partial orders. If $\mathbf{a}$ is a minimal Turing degree, then any non-total continuous degree below a would be quasi-minimal, hence there are none. This proves the following.

COROLlary 7.4. The minimal continuous degrees are exactly the minimal Turing degrees.

In particular, minimal continuous degrees exist. Gutteridge proved that there are no minimal enumeration degrees [11] (another proof was given by Cooper [7]). Therefore, the continuous degrees are not elementarily equivalent to the enumeration degrees. The same question is answered for the Turing degrees in Proposition 8.8, where we exhibit a natural elementary difference between the continuous degrees and the Turing degrees.

We turn to the proof of Theorem 7.2. We need a slight generalization of a result of V. P. Orevkov [24]. Working in the Russian school of constructive mathematics, Orevkov constructed a continuous retraction of the constructive points of the unit square $[0,1]^{2}$ onto its boundary $\partial\left([0,1]^{2}\right)$. In classical terms, his construction gives the following theorem.

TheOREM 7.5 (Orevkov, 1963). There is a partial computable $g:[0,1]^{2} \rightarrow$ $\partial\left([0,1]^{2}\right)$ defined for all computable points and fixing points on $\partial\left([0,1]^{2}\right)$.

The construction in the proof of Lemma 7.7 is essentially identical to Orevkov's (see Beeson [2] for a detailed presentation of a variant of this proof); we give it for the sake of completeness. It is well know that there is a nonempty $\Pi_{1}^{0}$ class which contains no computable elements [14]. Using this fact, we first produce a computable "singular covering" of (the computable reals in) $[-1,1]$.

LEMma 7.6. There is a computable sequence $\left\{J_{n}\right\}_{n \in \mathbb{N}}$ of closed rational intervals in $[-1,1]$ such that

(a) any two distinct $J_{n}$ intersect at most on an endpoint,

(b) $J=\bigcup_{n \in \mathbb{N}} J_{n}$ contains all computable reals in $[-1,1]$,

(c) if $x \in(-1,1)$ is an endpoint of $J_{n}$, then $x$ is also the endpoint of $J_{k}$ for some $k \neq n$, and

(d) $J$ is open (in the subspace topology on $[-1,1]$ ).

Proof. Associate to each $\sigma \in 2^{<\mathbb{N}}$ a closed rational interval $I_{\sigma} \subseteq[0,1]$ by successive bisection as follows. Let $I_{\emptyset}=[0,1]$. If $I_{\sigma}=\left[l_{\sigma}, r_{\sigma}\right]$ has already been defined, let $I_{\sigma^{\wedge} 0}=\left[l_{\sigma},\left(l_{\sigma}+r_{\sigma}\right) / 2\right]$ and $I_{\sigma \wedge 1}=\left[\left(l_{\sigma}+r_{\sigma}\right) / 2, r_{\sigma}\right]$. Note that $l_{\sigma}$

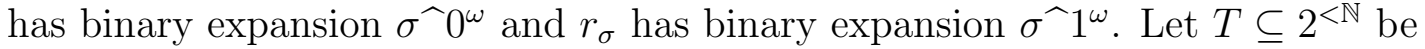


a computable infinite tree with no computable paths. Let $S=\left\{\sigma_{n}\right\}_{n \geq 1}$ be an enumeration without repetitions of all $\sigma \notin T$ such that $(\forall \tau \subset \sigma)[\tau \in T]$. Note that $S$ must be infinite because $T$ is infinite and has no computable paths. Set $J_{0}=[-1,0]$ and $J_{n}=I_{\sigma_{n}}$, for $n \geq 1$. Let $J=\bigcup_{n \in \mathbb{N}} J_{n}$.

Let $\sigma, \tau \in 2^{<\mathbb{N}}$ be distinct. If $I_{\sigma}$ and $I_{\tau}$ intersect at more than a single point, then either $\sigma \subset \tau$ or $\tau \subset \sigma$, so it is not possible to have both $\sigma$ and $\tau$ in $S$. This proves $(a)$. Take $x \in[-1,1]$. If $x<0$ then $x \in J$, so assume $x \in[0,1]$. Note that $x \notin J$ iff the binary expansion of $x$ is in $[T]$. The fact that $[T]$ contains no computable paths proves $(b)$. Now note that $0^{\omega} \notin[T]$, so $0^{m}=\sigma_{n} \in S$ for some $m, n \in \mathbb{N}$. Thus 0 is an endpoint of both $J_{0}$ and $J_{n}$. Next consider $l_{\sigma} \neq 0$, for some $\sigma \in S$. As noted above, $l_{\sigma}$ has binary expansion $\sigma^{\widehat{\Upsilon}} 0^{\omega}$. Let $b \in 2^{\mathbb{N}}$ be the other binary expansion of $l_{\sigma}$ (which is a binary rational). Because $b \notin[T]$ and $\sigma \not \subset b$, there must be a $\tau \neq \sigma$ such that $\tau \subset b$ and $\tau \in S$. If some binary expansion of $x$ begins with $\tau$, then $x \in I_{\tau}$. Therefore, $l_{\sigma} \in I_{\tau}$. Since $l_{\sigma} \in I_{\sigma} \cap I_{\tau}$, we must have $l_{\sigma}=r_{\tau}$ by $(a)$. The same argument holds if $r_{\sigma} \neq 1$, for some $\sigma \in S$. This proves $(c)$. Finally, $(d)$ follows from $(c)$.

Note that the degrees of reals in $[-1,1] \backslash J$ are exactly the same as the degrees of paths through $T$. We return to this observation in Section 8; for now, it is sufficient that $J$ contains the computable reals in $[-1,1]$.

Lemma 7.7. Let $x \in \mathbb{R}^{2}$ and $\varepsilon \in \mathbb{R}^{+}$be computable. There is a partial computable $g_{x, \varepsilon}: \mathbb{R}^{2} \rightarrow \mathbb{R}^{2} \backslash(x-\varepsilon, x+\varepsilon)^{2}$ which is defined for all computable points, fixes points on $\mathbb{R}^{2} \backslash(x-\varepsilon, x+\varepsilon)^{2}$, and maps points in $(x-\varepsilon, x+\varepsilon)^{2}$ to $\partial\left([x-\varepsilon, x+\varepsilon]^{2}\right)$. Moreover, we can compute $g_{x, \varepsilon}$ uniformly from $x$ and $\varepsilon$.

Proof. First we construct $g_{\langle 0,0\rangle, 1}$. Take $\left\{J_{n}\right\}_{n \in \mathbb{N}}$ from the previous lemma. Let $A_{n}=\bigcup_{k \leq n}\left(J_{n} \times J_{k} \cup J_{k} \times J_{n}\right)$. Define $B_{0}=\mathbb{R}^{2} \backslash(-1,1)^{2}$ and $B_{n+1}=B_{n} \cup$ $A_{n}$. We define a computable sequence $\left\{h_{n}\right\}_{n \in \mathbb{N}}$ of partial computable functions $h_{n}: \mathbb{R}^{2} \rightarrow \mathbb{R}^{2}$ such that range $\left(h_{n}\right)=B_{n}$ and $h_{n+1} \uparrow B_{n}=h_{n}$. Let $h_{0}$ be the identity on $B_{0}$. So $B_{n}=\left(\mathbb{R}^{2} \backslash(-1,1)^{2}\right) \cup\left(\bigcup_{k<n} J_{k}\right)^{2}$.

Suppose that $h_{n}$ has been defined. We must extend it to $A_{n}$. Note that $A_{n}$ is a finite union of rectangles properly contained in $C=J_{n} \times[-1,1] \cup[-1,1] \times J_{n}$ and that only the boundary of $C$ intersects $B_{n}$. Let $P_{1}, \ldots, P_{m}$ be the connected components of $A_{n}$. If $\partial P_{i} \cap \partial C=\emptyset$, then $P_{i}=C$. But this is impossible, because $A_{n} \supseteq P_{i}$ is not all of $C$; in particular, $J_{n} \times J_{n+1} \subset C$, but $J_{n} \times J_{n+1} \nsubseteq A_{n}$. Therefore, some open segment $s_{i}$ on the boundary of $P_{i}$ is in the interior of $C$; clearly $s_{i}$ is disjoint from $B_{n}$. In other words, the only part of $P_{i}$ on which $h_{n}$ might already be defined is $\partial P_{i} \backslash s_{i}$.

Now extend $h_{n}$ to $P_{i}$ as follows. First extend $h_{n}$ to all of $\partial P_{i} \backslash s_{i}$ such that $h_{n}\left[\partial P_{i} \backslash s_{i}\right] \subseteq \partial\left([-1,1]^{2}\right)$. This is possible because we can easily extend $h_{n}$ to any segment for which it is already defined on the endpoints. It is also easy to retract $P_{i}$ onto $\partial P_{i} \backslash s_{i}$ (because $P_{i}$ is simply connected). By composing the retraction with the extension of $h_{n}$, we define $h_{n+1}$ on $P_{i}$. Doing this for each $1 \leq i \leq m$ defines $h_{n+1}$ on $A_{n}$, as required. 
To compute $g_{\langle 0,0\rangle, 1}(z)$, search for a $n \in \mathbb{N}$ such that $z$ is in the interior of $B_{n}$. If such an $n$ is found, set $g_{\langle 0,0\rangle, 1}(z)=h_{n}(z)$. Therefore, $g_{\langle 0,0\rangle, 1}: \mathbb{R}^{2} \rightarrow \mathbb{R}^{2} \backslash(-1,1)^{2}$ is a partial computable function which fixes points on $\mathbb{R}^{2} \backslash(-1,1)^{2}$, and maps points in $(-1,1)^{2}$ to $\partial\left([-1,1]^{2}\right)$. Moreover, $g_{\langle 0,0\rangle, 1}$ is defined for all points in the interior of some $B_{n}$. These are exactly the points in $\left(\mathbb{R}^{2} \backslash[-1,1]^{2}\right) \cup J^{2}$, where $J=\bigcup_{n \in \mathbb{N}} J_{n}$ (because the endpoints of $J_{i}$ are in the interior of $\bigcup_{n \leq j} J_{n}$, for large enough $j$ ). Therefore, $g_{\langle 0,0\rangle, 1}(z)$ is defined for all computable $z \in \mathbb{R}^{2}$.

Finally, if $x \in \mathbb{R}^{2}$ and $\varepsilon \in \mathbb{R}^{+}$are computable, define $g_{x, \varepsilon}(z)=\varepsilon g_{\langle 0,0\rangle, 1}((z-$ $x) / \varepsilon)+x$. Note that $g_{x, \varepsilon}$ has the required properties. The construction is clearly uniform in $x$ and $\varepsilon$.

In the next proof, we use the fact that $g_{x, \varepsilon}$ moves every point in $\mathbb{R}^{2}$ less than $2 \sqrt{2} \varepsilon$, while also ensuring that no point in the range is within less than $\varepsilon$ of $x$.

Proposition 7.8. There is a partial computable function $f:[0,1]^{\mathbb{N}} \rightarrow[0,1]^{2}$ such that $f$ is defined on all sequences of computable reals and if $f(\alpha) \downarrow=\left\langle a_{0}, a_{1}\right\rangle$, then either $a_{0} \notin$ range $(\alpha)$ or $a_{1} \notin \operatorname{range}(\alpha)$.

Proof. We inductively define a sequence $\left\{f^{n}\right\}_{n \in \mathbb{N}}$ of functions $f^{n}:[0,1]^{\mathbb{N}} \rightarrow$ $[0,1]^{2}$. Let $\alpha \in[0,1]^{\mathbb{N}}$ and set $f^{0}(\alpha)=\langle 1 / 2,1 / 2\rangle$. Assume that we have already defined $f^{n}(\alpha)$. Let

$$
f^{n+1}(\alpha)=g_{\langle\alpha(j), \alpha(k)\rangle, 4^{-n-1} / 2}\left(f^{n}(\alpha)\right)
$$

where $n=\langle j, k\rangle$. Define $f(\alpha)=\lim _{n \rightarrow \infty} f^{n}(\alpha)$. It remains to show that $f$ satisfies the theorem.

First fix $\alpha \in[0,1]^{\mathbb{N}}$ and assume that $f^{n}(\alpha) \downarrow$ for all $n$. We know that, for $n=\langle j, k\rangle$, the function $g_{\langle\alpha(j), \alpha(k)\rangle, 4^{-n-1} / 2}$ moves each point by less than $\sqrt{2} 4^{-n-1}$. Hence $f(\alpha)=\lim _{n \rightarrow \infty} f^{n}(\alpha)$ exists and, in fact,

$$
\left|f(\alpha)-f^{n}(\alpha)\right|<\sum_{i=n}^{\infty} \sqrt{2} 4^{-i-1}=\frac{\sqrt{2}}{3} 4^{-n} .
$$

In particular, $|f(\alpha)-\langle 1 / 2,1 / 2\rangle|<\sqrt{2} / 3<1 / 2$, so $f(\alpha) \in[0,1]^{2}$. Also note that $f(\alpha)$ is computable from the sequence $\left\{f^{n}(\alpha)\right\}_{n \in \mathbb{N}}$. But $\left\{f^{n}(\alpha)\right\}_{n \in \mathbb{N}}$ is computable uniformly from $\alpha$, so $f:[0,1]^{\mathbb{N}} \rightarrow[0,1]^{2}$ is a partial computable function.

Now fix $\alpha \in[0,1]^{\mathbb{N}}$ with $f(\alpha) \downarrow=\left\langle a_{0}, a_{1}\right\rangle$. Assume, for a contradiction, that both $a_{0} \in \operatorname{range}(\alpha)$ and $a_{1} \in \operatorname{range}(\alpha)$. In particular, assume that $\left\langle a_{0}, a_{1}\right\rangle=$ $\langle\alpha(j), \alpha(k)\rangle$. Consider $f^{n+1}(\alpha)$, where $n=\langle j, k\rangle$. It follows from the definition of $g_{\langle\alpha(j), \alpha(k)\rangle, 4^{-n-1} / 2}$ that $\left|\langle\alpha(j), \alpha(k)\rangle-f^{n+1}(\alpha)\right| \geq 4^{-n-1} / 2$. But from above, we know that $\left|f(\alpha)-f^{n+1}(\alpha)\right|<(\sqrt{2} / 3) 4^{-n-1}$. Therefore,

$$
\begin{aligned}
|\langle\alpha(j), \alpha(k)\rangle-f(\alpha)| & \geq\left|\langle\alpha(j), \alpha(k)\rangle-f^{n+1}(\alpha)\right|-\left|f(\alpha)-f^{n+1}(\alpha)\right| \\
& >\frac{1}{2} 4^{-n-1}-\frac{\sqrt{2}}{3} 4^{-n-1}>0 .
\end{aligned}
$$

But this is a contradiction. So either $a_{0} \notin \operatorname{range}(\alpha)$ or $a_{1} \notin \operatorname{range}(\alpha)$. 
Finally, assume that $\alpha \in[0,1]^{\mathbb{N}}$ is a sequence of computable reals. We show, by induction on $n$, that $f^{n}(\alpha)$ converges to a computable point for each $n$. Assume that $f^{n}(\alpha)$ is defined and is computable. Let $n=\langle j, k\rangle$; the function $g_{\langle\alpha(j), \alpha(k)\rangle, 4^{-n-1} / 2}$ is partial computable and converges on computable points. Thus, $f^{n+1}(\alpha)=g_{\langle\alpha(j), \alpha(k)\rangle, 4^{-n-1} / 2}\left(f^{n}(\alpha)\right)$ exists. Also, the image of a computable point under a partial computable function is computable, so $f^{n+1}(\alpha)$ is computable. Therefore, $f^{n}(\alpha)$ exists for all $n$. This implies that $f(\alpha)$ converges.

We have all but finished the proof of Theorem 7.2.

Proof of Theorem 7.2. Consider the partial computable coordinate functions $f_{0}$ and $f_{1}$ of the function $f:[0,1]^{\mathbb{N}} \rightarrow[0,1]^{2}$ constructed in Proposition 7.8. If $\alpha \in[0,1]^{\mathbb{N}}$ is a sequence of computable reals, then $f(\alpha) \downarrow$ and either $f_{0}(\alpha) \notin$ range $(\alpha)$ or $f_{1}(\alpha) \notin$ range $(\alpha)$. Therefore, $\alpha$ is diagonalized by either $f_{0}$ or $f_{1}$.

This proves that two partial computable functions suffice to diagonalize all sequences in $[0,1]^{\mathbb{N}}$ of computable reals. It is worth pointing out that no single partial computable function can diagonalize all such sequences. To see this, assume that $g:[0,1]^{\mathbb{N}} \rightarrow[0,1]$ is a partial computable function which converges on sequences of computable reals. Let $\alpha_{x}=(x, 0,0, \ldots)$ and define a partial computable function $h:[0,1] \rightarrow[0,1]$ by $h(x)=g\left(\alpha_{x}\right)$. Note that $h$ must converge on all computable reals in $[0,1]$. Therefore, $h$ has a computable fixed point $^{8} c \in[0,1]$. So $g\left(\alpha_{c}\right)=c \in$ range $\left(\alpha_{c}\right)$, proving that $g$ does not diagonalize all sequences of computable reals.

§8. Intervals containing a non-total continuous degree. We have not fully exploited the constructions of the previous two sections. A more careful analysis of these constructions - or more exactly, of relativizations of these constructions - will provide significant insight into the relationship of the continuous degrees to the Turing degrees. We begin with a review of the necessary computability theory. If $T \subseteq 2^{<\mathbb{N}}$ is a tree computable in a Turing degree $\mathbf{b}$, then $[T]$ is called a $\Pi_{1}^{0}(\mathbf{b})$ class.

Definition 8.1. If $\mathbf{a}$ and $\mathbf{b}$ are Turing degrees, then $\mathbf{a}$ is a $P A$ degree relative to $\mathbf{b}(\mathbf{a} \gg \mathbf{b})$ if every nonempty $\Pi_{1}^{0}(\mathbf{b})$ class contains a path computable from a. For $A, B \subseteq \mathbb{N}$, we write $A \gg B$ to mean that $\operatorname{deg}_{T}(A) \gg \operatorname{deg}_{T}(B)$.

Simpson discusses this relation in [36]. A degree $\mathbf{b} \gg \mathbf{0}$ is called a PA degree. It follows from work of Scott [34] and Solovay (unpublished) that the PA degrees are exactly the degrees of complete consistent extensions of Peano arithmetic. Note that the collection of such extensions is a $\Pi_{1}^{0}$ class, so there is a nonempty $\Pi_{1}^{0}$ class containing only members of PA degree. The proof of Lemma 8.3 requires the relativization of this observation: for every Turing degree $\mathbf{b}$, there exists a

\footnotetext{
${ }^{8}$ This follows from the intermediate value theorem for partial computable functions which converge on the computable reals. That, in turn, follows from the classical bisection proof.
} 
nonempty $\Pi_{1}^{0}(\mathbf{b})$ class containing only members of PA degree relative to $\mathbf{b}$. A well-known example is the class

$$
D N C_{2}^{B}=\left\{f \in 2^{\mathbb{N}} \mid(\forall e \in \mathbb{N})\left[f(e) \neq \varphi_{e}^{B}(e)\right]\right\},
$$

of diagonally not $B$-computable $\{0,1\}$-valued functions, where $B \subseteq \mathbb{N}$ has degree b. The proof that every element of $D N C_{2}^{B}$ has $\mathrm{PA}$ degree relative to $\mathbf{b}$ is straightforward. In fact, the PA degrees relative to $\mathbf{b}$ are exactly the degrees of members of $D N C_{2}^{B}$.

The main result of this section is Corollary 8.5, which characterizes the total degrees $\mathbf{b}<\mathbf{a}$ between which there is a non-total degree as the intervals $\mathbf{b} \ll \mathbf{a}$. For one direction, we relativize the effective content of Section 6 .

THEOREM 8.2. If $\mathbf{a}$ and $\mathbf{b}$ are total degrees and $\mathbf{b} \ll \mathbf{a}$, then there is a nontotal degree $\mathbf{v}$ with $\mathbf{b}<\mathbf{v}<\mathbf{a}$.

Proof. Recall that the multivalued function $\Psi:[0,1]^{\mathbb{N}} \rightarrow[0,1]^{\mathbb{N}}$ constructed in the proof of Lemma 6.2 has the property that $\{A \oplus B \subseteq \mathbb{N} \mid \Pi(B) \in \Psi(\Pi(A))\}$ is a $\Pi_{1}^{0}$ class, where $\Pi: 2^{\mathbb{N}} \rightarrow[0,1]^{\mathbb{N}}$ is the function taking b-representations to the sequences that they represent. Choose $b \in[0,1]$ of degree $\mathbf{b}$ and define a new multivalued function $\Psi_{b}:[0,1]^{\mathbb{N}} \rightarrow[0,1]^{\mathbb{N}}$ by

$$
\Psi_{b}(\alpha)(n)= \begin{cases}b, & \text { if } n=0 \\ \Psi(\alpha)(n-1), & \text { if } n>0\end{cases}
$$

It follows that $\left\{A \oplus B \subseteq \mathbb{N} \mid \Pi(B) \in \Psi_{b}(\Pi(A))\right\}$ is a $\Pi_{1}^{0}(\mathbf{b})$ class. Of course, $\{A \oplus A \mid A \subseteq \mathbb{N}\}$ is a $\Pi_{1}^{0}$ class, so the intersection $\mathcal{G}=\{A \oplus A \subseteq \mathbb{N} \mid \Pi(A) \in$ $\left.\Psi_{b}(\Pi(A))\right\}$ is a $\Pi_{1}^{0}(\mathbf{b})$ class. Theorem 6.1 implies that $\Psi_{b}$ has a fixed point, so $\mathcal{G}$ is nonempty. Therefore, there is some a-computable $A \oplus A \in \mathcal{G}$. Let $\alpha=\Pi(A)$ and $\mathbf{v}=\operatorname{deg}_{r}(\alpha)$. So $\mathbf{v} \leq \mathbf{a}$. Note that $\alpha \in \Psi_{b}(\alpha)$. As in Theorem 6.3, the fixed points of $\Psi_{b}$ are not computably diagonalizable and hence, by Proposition 5.3, have non-total degree. Therefore, $\mathbf{v}$ is non-total. Finally, $\alpha(0)=b$ because $\alpha$ is in the image of $\Psi_{b}$. So $\mathbf{b} \leq \mathbf{v}$.

We now relativize the results of Section 7 . Let $\mathbf{b}$ be a Turing degree. We say that a sequence $\alpha \in[0,1]^{\mathbb{N}}$ is b-computably diagonalizable if there is an $x \in[0,1]$ such that $x$ has degree $\leq \operatorname{deg}_{r}(\alpha) \cup \mathbf{b}$ and $x \notin \operatorname{range}(\alpha)$. Theorem 7.2 states that sequences of computable reals are computably diagonalizable. We generalize this result and give a sufficient condition for a sequence to be b-computably diagonalizable.

LEMmA 8.3. Let $\mathcal{I}$ be a countable ideal in the Turing degrees and $\mathbf{b}$ a Turing degree such that $\mathbf{c} \in \mathcal{I}$ implies that $\mathbf{b} \nless \mathbf{c}$. Then every sequence $\alpha \in[0,1]^{\mathbb{N}}$ with $\operatorname{deg}_{T}\left(\alpha_{i}\right) \in \mathcal{I}$, for all $i \in \mathbb{N}$, is b-computably diagonalizable.

Proof. Let $T \subseteq 2^{<\mathbb{N}}$ be any tree (computable or not) such that $[T]$ is nonempty and contains no computable elements. Apply the construction in Lemma 7.6 to produce a sequence $\left\{J_{n}\right\}_{n \in \mathbb{N}}$ of closed rational intervals in $[-1,1]$. 
Let $J=\bigcup_{n \in \mathbb{N}} J_{n}$. As noted previously, the reals in $[-1,1] \backslash J$ have the same degrees as the paths through $[T]$. Now take $T$ computable in $\mathbf{b}$ such that the $\Pi_{1}^{0}(\mathbf{b})$ class $[T]$ is nonempty and contains only elements of degree PA relative to b. Then the sequence $\left\{J_{n}\right\}_{n \in \mathbb{N}}$ is computable in $\mathbf{b}$ and $J$ contains all reals in $[-1,1]$ with degree in $\mathcal{I}$ (none of which are PA relative to $\mathbf{b}$ ); the other conditions of Lemma 7.6 are unchanged.

Now use $\left\{J_{n}\right\}_{n \in \mathbb{N}}$ in the construction of $g_{\langle 0,0\rangle, 1}: \mathbb{R}^{2} \rightarrow \mathbb{R}^{2} \backslash(x-1, x+1)^{2}$ as in Lemma 7.7. Then $g_{\langle 0,0\rangle, 1}$ is partial computable relative to $\mathbf{b}$ and is defined on all points with degree in $\mathcal{I}$. Recall that if $x \in \mathbb{R}^{2}$ and $\varepsilon \in \mathbb{R}^{+}$, we define $g_{x, \varepsilon}(z)=\varepsilon g_{\langle 0,0\rangle, 1}((z-x) / \varepsilon)+x$. As before, $g_{x, \varepsilon}: \mathbb{R}^{2} \rightarrow \mathbb{R}^{2} \backslash(x-\varepsilon, x+\varepsilon)^{2}$ fixes points on $\mathbb{R}^{2} \backslash(x-\varepsilon, x+\varepsilon)^{2}$, and maps points in $(x-\varepsilon, x+\varepsilon)^{2}$ to $\partial\left([x-\varepsilon, x+\varepsilon]^{2}\right)$. Assume that $\operatorname{deg}_{T}(x), \operatorname{deg}_{T}(\varepsilon) \in \mathcal{I}$. Then if $z \in \mathbb{R}^{2}$ has degree in $\mathcal{I}$, so does $(z-x) / \varepsilon$. Therefore, $g_{x, \varepsilon}$ is defined on all points with degree in $\mathcal{I}$. Furthermore, $g_{x, \varepsilon}(z)$ is computable from $x \oplus \varepsilon \oplus z$ and $\mathbf{b}$ (with all possible uniformity). In particular, $g_{x, \varepsilon}(z)$ has degree in $\mathcal{I}$ whenever $z \in \mathbb{R}^{2}$ does.

Next we inductively define a sequence $\left\{f^{n}\right\}_{n \in \mathbb{N}}$ of functions $f^{n}:[0,1]^{\mathbb{N}} \rightarrow[0,1]^{2}$ as in Proposition 7.8. For $\alpha \in[0,1]^{\mathbb{N}}$ set $f^{0}(\alpha)=\langle 1 / 2,1 / 2\rangle$ and

$$
f^{n+1}(\alpha)=g_{\langle\alpha(j), \alpha(k)\rangle, 4^{-n-1} / 2}\left(f^{n}(\alpha)\right),
$$

where $n=\langle j, k\rangle$. Assume $\operatorname{deg}_{T}\left(\alpha_{i}\right) \in \mathcal{I}$, for all $i \in \mathbb{N}$. Then by induction on $n$, $f^{n}(\alpha) \downarrow$ and $\operatorname{deg}_{T}\left(f^{n}(\alpha)\right) \in \mathcal{I}$. Define $f(\alpha)=\lim _{n \rightarrow \infty} f^{n}(\alpha)$. By the same arguments as before, $f(\alpha) \downarrow \in[0,1]^{2}$ and if $f(\alpha)=\left\langle a_{0}, a_{1}\right\rangle$, then either $a_{0} \notin \operatorname{range}(\alpha)$ or $a_{1} \notin \operatorname{range}(\alpha)$. Without loss of generality, assume $a_{0} \notin \operatorname{range}(\alpha)$. Also as before, $f(\alpha)$ is computable from the sequence $\left\{f^{n}(\alpha)\right\}_{n \in \mathbb{N}}$. But $\left\{f^{n}(\alpha)\right\}_{n \in \mathbb{N}}$ is computable with respect to $\alpha$ and $\mathbf{b}$, so $f(\alpha)$ has degree $\leq \operatorname{deg}_{r}(\alpha) \cup \mathbf{b}$. Because $a_{0} \leq_{r} f(\alpha)$, we have that $\alpha$ is b-computably diagonalizable. But $\alpha \in[0,1]^{\mathbb{N}}$ was an arbitrary sequence of reals with degrees in $\mathcal{I}$.

Given a continuous degree $\mathbf{v}$, we define the Turing ideal below $\mathbf{v}$ by $\mathcal{I}_{T}(\mathbf{v})=$ $\{\mathbf{a} \leq \mathbf{v} \mid \mathbf{a}$ total $\}$. As the name suggests, $\mathcal{I}_{T}(\mathbf{v})$ can be viewed as a countable ideal in the Turing degrees.

THEOREM 8.4. If $\mathbf{v}$ is a non-total continuous degree and $\mathbf{b}<\mathbf{v}$ is total, then there is a total degree $\mathbf{c}$ with $\mathbf{b} \ll \mathbf{c}<\mathbf{v}$.

Proof. Let $\mathbf{v}$ be a continuous degree and assume that there is a total degree $\mathbf{b}<\mathbf{v}$ such that if $\mathbf{c}<\mathbf{v}$ is total, then $\mathbf{c} \ngtr \mathbf{b}$. We must prove that $\mathbf{v}$ is also total. Let $\mathcal{I}=\mathcal{I}_{T}(\mathbf{v})$. Then $\mathcal{I}$ and $\mathbf{b}$ satisfy the hypotheses of Lemma 8.3. Take an arbitrary sequence $\alpha \in[0,1]^{\mathbb{N}}$ of degree $\mathbf{v}$. Every coordinate of $\alpha$ has degree in $\mathcal{I}$, so by the lemma, there is an $x \in[0,1]$ such that $x$ has degree $\leq \mathbf{d e g}_{r}(\alpha) \cup \mathbf{b}$ and $x \notin \operatorname{range}(\alpha)$. But $\mathbf{b} \leq \operatorname{deg}_{r}(\alpha)=\mathbf{v}$. Therefore $x$ has degree $\leq \mathbf{v}$, so $\alpha$ is computably diagonalizable. This proves that every sequence in $\mathbf{v}$ is computably diagonalizable. Thus by Proposition 5.3, $\mathbf{v}$ is total.

Corollary 8.5. Let $\mathbf{a}$ and $\mathbf{b}$ be total degrees. Then $\mathbf{b} \ll \mathbf{a}$ iff there is $a$ non-total degree $\mathbf{v}$ with $\mathbf{b}<\mathbf{v}<\mathbf{a}$. 
Proof. One direction was already proved in Theorem 8.2. For the other direction, assume that there is a non-total $\mathbf{v}$ such that $\mathbf{b}<\mathbf{v}<\mathbf{a}$. By Theorem 8.4, there is a total degree $\mathbf{c}$ with $\mathbf{b} \ll \mathbf{c}<\mathbf{v}$. So $\mathbf{c} \leq \mathbf{a}$, but $\mathbf{b} \ll \mathbf{c} \leq \mathbf{a}$ implies that $\mathbf{b} \ll \mathbf{a}$ (every $\Pi_{1}^{0}(\mathbf{b})$ class has a member computable from $\mathbf{c}$, hence from a). This completes the proof.

Now that we know where non-total degrees occur, certain results for the continuous degrees can be proved using facts about the Turing degrees. This allows us to benefit from the large body of existing work, although direct proofs could avoid some of the machinery implicit in the results cited below.

We provide two examples. Fix a non-computable c.e. degree $\mathbf{a}_{0} \ngtr \mathbf{0}$. The existence of such a degree follows from the Arslanov completeness criterion [1]; in fact, $\mathbf{0}^{\prime}$ is the only c.e. PA degree. Note that the continuous degrees between $\mathbf{0}$ and $\mathbf{a}_{0}$ are exactly the Turing degrees in this interval.

Proposition 8.6. The continuous degrees do not form a lattice.

Proof. Using the Sacks Density Theorem [32] repeatedly, produce an increasing sequence of c.e. degrees $\mathbf{b}_{0}<\mathbf{b}_{1}<\mathbf{b}_{2}<\cdots<\mathbf{a}_{0}$, where $\mathbf{a}_{0}$ is from the preceding lemma. Choose $\mathbf{c}$ and $\mathbf{d}$ be an exact pair for $\left\{\mathbf{b}_{n}\right\}_{n \in \mathbb{N}}$ in the Turing degrees [39]. In other words, for all total degrees $\mathbf{e}, \mathbf{e} \leq \mathbf{c}$ and $\mathbf{e} \leq \mathbf{d}$ iff $(\exists n)\left[\mathbf{e} \leq \mathbf{b}_{n}\right]$. Assume, for a contradiction, that $\mathbf{v}=\mathbf{c} \cap \mathbf{d} \cap \mathbf{a}_{0}$ in the continuous degrees. Because $\mathbf{v} \leq \mathbf{a}_{0}$, we know that $\mathbf{v}$ is total. Also $\mathbf{v}$ is below both $\mathbf{c}$ and $\mathbf{d}$, so $\mathbf{v} \leq \mathbf{b}_{n}$ for some $n \in \mathbb{N}$. But then $\mathbf{b}_{n+1} \leq \mathbf{c} \cap \mathbf{d} \cap \mathbf{a}_{0}=\mathbf{v} \leq \mathbf{b}_{n}$, which is a contradiction.

Simpson proved that the first order theory of the Turing degrees is equivalent to second order arithmetic [37]. Slaman and Woodin proved the corresponding theorem for the enumeration degrees [38]. It should come as no surprise that the first order theory of the continuous degrees has the same complexity; this is verified in our second example. It is convenient to introduce some notation for initial segments of the Turing degrees and the continuous degrees. If $\mathbf{b}$ is a Turing degree, then define $[\mathbf{0}, \mathbf{b}]_{T}=\mathcal{I}_{T}(\mathbf{b})=\{\mathbf{a} \leq \mathbf{b} \mid \mathbf{a}$ total $\}$. For $\mathbf{v}$ continuous, let $[\mathbf{0}, \mathbf{v}]_{r}=\{\mathbf{w} \leq \mathbf{v} \mid \mathbf{w}$ continuous $\}$.

Proposition 8.7. The first order theory of the continuous degrees (as a partial order) is equivalent to the second order theory of arithmetic.

Proof Sketch. Interpreting the theory of the continuous degrees in second order arithmetic is routine. For the other direction, we use a method introduced by Nerode and Shore to translate second order arithmetic into the theory of the Turing degrees [23]. They encode structures as computably presentable countable distributive lattices; let $\mathcal{L}$ encode the standard model of arithmetic. Two facts about the continuous degrees must be verified to apply the translation.

(i) There is a continuous degree $\mathbf{v}$ such that $\mathcal{L}$ is isomorphic $[\mathbf{0}, \mathbf{v}]_{r}$.

(ii) If $\mathbf{w}$ is a continuous degree such that $[\mathbf{0}, \mathbf{w}]_{r}$ is a distributive lattice and $\mathcal{B} \subseteq[\mathbf{0}, \mathbf{w}]_{r}$ is a collection of minimal degrees, then there are continuous degrees $\mathbf{y}$ and $\mathbf{z}$ such that $(\forall$ minimal $\mathbf{b})[\mathbf{b} \in \mathcal{B} \Longleftrightarrow(\mathbf{b} \leq \mathbf{y} \wedge \mathbf{b} \leq \mathbf{z})]$. 
The elements of an encoded structure are represented by minimal degrees, so (ii) allows us to interpret second order quantification (which also allows us to recognize the standard model of arithmetic). The details of the translation can be found in [23] and [19].

We verify the two conditions. Lerman proved in [19] that if a is a noncomputable c.e. degree, then there is a $\mathbf{d} \leq \mathbf{a}$ such that $\mathcal{L}$ is isomorphic to $[\mathbf{0}, \mathbf{d}]_{T}$ (in fact, $\mathcal{L}$ can be replaced by any $\mathbf{0}^{\prime}$ presentable upper semi-lattice with least element). Apply this theorem to a non-computable c.e. degree $\mathbf{a}_{0} \gg \mathbf{0}$ below which all continuous degrees are total-to get a $\mathbf{v} \leq \mathbf{a}_{0}$ such that $\mathcal{L}$ is isomorphic to $[\mathbf{0}, \mathbf{v}]_{T}=[\mathbf{0}, \mathbf{v}]_{r}$. This proves (i).

Now take $\mathbf{w}$ and $\mathcal{B}$ as in (ii). Let $\mathcal{I}_{r}$ be the ideal generated by $\mathcal{B}$ in the continuous degrees. Because $[\mathbf{0}, \mathbf{w}]_{r}$ is a distributive lattice, all minimal degrees in $\mathcal{I}_{r}$ are in $\mathcal{B}$. Let $\mathcal{I}$ be the total degrees in $\mathcal{I}_{r}$. Note that $\mathcal{I}$ is an ideal in the Turing degrees and that $\mathcal{B} \subseteq \mathcal{I}$. Let $\mathbf{y}$ and $\mathbf{z}$ be an exact pair for $\mathcal{I}$ in the Turing degrees. Because every minimal degree $\mathbf{b}$ is total, $\mathbf{b} \in \mathcal{B}$ iff $\mathbf{b} \leq \mathbf{y}$ and $\mathbf{b} \leq \mathbf{z}$. This proves (ii), so the translation of second order arithmetic given in [23] works for the continuous degrees as well.

In Section 7, we saw that the existence of a minimal degree distinguishes the continuous degrees from the enumeration degrees, but we have not yet seen an elementary difference between the continuous degrees and the Turing degrees. We finish this section with a natural example of such a difference. First, note that there is significant agreement between the theories of the two structures. In particular, the previous proof shows that the same translation used in [23] to interpret second order arithmetic into the first order theory of the Turing degrees works also for the continuous degrees.

Proposition 8.8. The continuous degrees are not elementarily equivalent to the Turing degrees.

Proof. Let $\varphi$ be the first order sentence

$$
(\exists \mathbf{a})(\forall \mathbf{b} \geq \mathbf{a})\left(\exists \mathbf{c}_{0}, \mathbf{c}_{1}<\mathbf{b}\right)\left[\mathbf{b}=\mathbf{c}_{0} \cup \mathbf{c}_{1} \wedge \mathbf{0}=\mathbf{c}_{0} \cap \mathbf{c}_{1}\right]
$$

In words, $\varphi$ states that there is a cone of degrees which are each the join of a minimal pair. Note that meet and join are definable in the order, so $\varphi$ can be expressed in the language of partial orders. We claim that $\varphi$ is true for the Turing degrees but false for the continuous degrees. For the Turing degrees, $\varphi$ is satisfied by $\mathbf{a}=\mathbf{0}^{\prime}$. In fact, Posner [25] proved that for every $\mathbf{b} \geq \mathbf{0}^{\prime}$, the degrees below $\mathbf{b}$ are complemented. In other words, for any $\mathbf{c}_{0}<\mathbf{b}$, there is a $\mathbf{c}_{1}$ such that $\mathbf{b}=\mathbf{c}_{0} \cup \mathbf{c}_{1}$ and $\mathbf{c}_{0}$ and $\mathbf{c}_{1}$ form a minimal pair.

Now consider the continuous degrees. By Theorem 8.2, every cone in the continuous degrees contains a non-total degree. Therefore, to show that $\varphi$ fails for the continuous degrees it is sufficient to prove that the join of a minimal pair of continuous degrees must be total. 
Take two sequences $\alpha_{0}, \alpha_{1} \in[0,1]^{\mathbb{N}}$ which form a minimal pair in the continuous degrees. In particular, neither is computable and there is no noncomputable total degree below both. By Corollary 7.3, there are non-computable reals $x_{0}, x_{1} \in[0,1]$ such that $x_{0} \leq_{r} \alpha_{0}$ and $x_{1} \leq_{r} \alpha_{1}$. But then $x_{0} \leq_{r} \alpha_{1}$ and $x_{1} \not \underline{x}_{r} \alpha_{0}$. We claim that $x_{0} \oplus \alpha_{1}$ has total degree. To see this, define a new sequence $\beta \in[0,1]^{\mathbb{N}}$ by $\beta(i)=\left(\alpha_{1}(i)+x_{0}\right) / 2$. Then $\beta$ contains no binary rationals, since $x_{0} \not_{r} \alpha_{1}$. So by Lemma $5.2, \beta$ has total degree. Therefore, $x_{0} \oplus \alpha_{1} \equiv_{r} x_{0} \oplus \beta$ has total degree (the join of total degrees is total). By the same argument, $x_{1} \oplus \alpha_{0}$ must have total degree. But then $\alpha_{0} \oplus \alpha_{1} \equiv_{r}\left(x_{0} \oplus \alpha_{0}\right) \oplus\left(x_{1} \oplus \alpha_{1}\right) \equiv_{r}\left(x_{0} \oplus \alpha_{1}\right) \oplus\left(x_{1} \oplus \alpha_{0}\right)$ also has total degree. Therefore, every minimal pair of continuous degrees has total join, completing the proof.

§9. The ideal below a non-total continuous degree. Recall from the last section that if $\mathbf{v}$ is a continuous degree, then the Turing ideal below $\mathbf{v}$ is $\mathcal{I}_{T}(\mathbf{v})=\{\mathbf{a} \leq \mathbf{v} \mid$ a total $\}$. Note that if $\alpha \in[0,1]^{\mathbb{N}}$ is not computably diagonalizable, then $\mathcal{I}_{T}\left(\mathbf{d e g}_{r}(\alpha)\right)=\left\{\mathbf{d e g}_{T}\left(\alpha_{i}\right)\right\}_{i \in \mathbb{N}}$. Our first goal in this section is to characterize the Turing ideals below non-total continuous degrees as the Scott ideals. A nonempty countable class $\mathcal{S} \subseteq 2^{\mathbb{N}}$ is called a Scott set if

(a) $A, B \in \mathcal{S}$ implies that $A \oplus B \in \mathcal{S}$

(b) $A \in \mathcal{S}$ and $B \leq_{T} A$ implies $B \in \mathcal{S}$, and

(c) for every $A \in \mathcal{S}$, there is a $B \in \mathcal{S}$ such that $B \gg A$.

This notion is classical; Scott proved that $\mathcal{S} \subseteq 2^{\mathbb{N}}$ is a Scott set iff it is the field of (standard initial segments of) sets arithmetically definable in some complete extension of Peano arithmetic [34]. If $\mathcal{I}$ is a countable ideal in the Turing degrees, we call $\mathcal{I}$ a $S$ cott ideal if $\mathbf{a} \in \mathcal{I}$ implies that there is $\mathbf{a} \mathbf{b} \in \mathcal{I}$ with $\mathbf{b} \gg \mathbf{a}$. Note that a Scott ideal is just the collection of Turing degrees of elements of a Scott set. By Theorem 8.4, if $\mathbf{v}$ is non-total, then $\mathcal{I}_{T}(\mathbf{v})$ is a Scott ideal. In other words, if $f \in \mathcal{C}[0,1]$ has non-total degree, then the collection of $f$-computable subsets of $\mathbb{N}$ is a Scott set. Conversely, every Scott set is represented in this way; by Theorem 9.3, the Scott ideals are exactly the ideals below non-total continuous degrees. In fact, by Theorem 9.5 , if $\mathcal{I}$ is a Scott ideal, then there are $2^{\aleph_{0}}$ pairwise incomparable continuous degrees $\mathbf{v}$ such that $\mathcal{I}=\mathcal{I}_{T}(\mathbf{v})$. We finish the article by proving that if $\mathbf{v}$ is any non-total continuous degree, then there is another degree $\mathbf{w} \mid \mathbf{v}$ with the same Turing ideal.

To prove these theorems we must be able to control the construction of sequences which are not computably diagonalizable, in particular, sequences which are fixed points of the multivalued function $\Psi:[0,1]^{\mathbb{N}} \rightarrow[0,1]^{\mathbb{N}}$ from Section 6 . Two lemmas will be useful. By the first lemma, we may construct such sequences in stages by "finite extensions". The second lemma tells us that at any stage of such a construction there is a coordinate whose value is unconstrained. 
Lemma 9.1. Let $\alpha \in[0,1]^{\mathbb{N}}$ be the union of a sequence $\alpha_{0} \subseteq \alpha_{1} \subseteq \alpha_{2} \subseteq$. . of partial functions $\alpha_{s}: \mathbb{N} \rightarrow[0,1]$. If each $\alpha_{s}$ can be extended to a fixed point of $\Psi$, then $\alpha$ is a fixed point of $\Psi$.

Proof. This is immediate. For each $n$, let $\widehat{\alpha}_{n} \in[0,1]^{\mathbb{N}}$ be an extension of $\alpha_{n}$ to a fixed point of $\Psi$. Note that $\lim _{n \rightarrow \infty} \widehat{\alpha}_{n}=\alpha$. For each $n$, we know that $\left\langle\widehat{\alpha}_{n}, \widehat{\alpha}_{n}\right\rangle$ is in the graph of $\Psi$ because $\widehat{\alpha}_{n}$ is a fixed point of $\Psi$. But the graph of $\Psi$ is closed, so $\langle\alpha, \alpha\rangle$ is in the graph of $\Psi$. So $\alpha$ is a fixed point of $\Psi$.

The second lemma deserves considerably more attention. Recall from Section 6 that $\Pi: 2^{\mathbb{N}} \rightarrow[0,1]^{\mathbb{N}}$ is the computable function taking every b-representation to the sequence which it represents.

Lemma 9.2. Let $\mathcal{G} \subseteq 2^{\mathbb{N}}$ be a $\Pi_{1}^{0}$ class. Let $\alpha_{0}: \mathbb{N} \rightarrow[0,1]$ be a partial sequence with finite support which can be extended to a fixed point of $\Psi$ in $\Pi[\mathcal{G}]$. Then there is an index $e \in \mathbb{N}$ such that for any $x \in[0,1]$, there is an extension of $\alpha_{0}$ to a fixed point $\alpha$ of $\Psi$ such that $\alpha \in \Pi[\mathcal{G}]$ and $\alpha(e)=x$.

Proof. Let Fix $_{\mathcal{G}}=\left\{\alpha \in[0,1]^{\mathbb{N}} \mid \alpha \in \Psi(\alpha) \cap \Pi[\mathcal{G}]\right\}$. We will define an index $e \in \mathbb{N}$ which attempts to prevent any extension of $\alpha_{0}$ from being in FIX , $_{\text {, }}$ contrary to assumption. We start with an informal description, the construction is given in more detail below. By the recursion theorem, we can find $e \in \mathbb{N}$ such that, if $\lambda$ is a representation of an extension $\beta \in[0,1]^{\mathbb{N}}$ of $\alpha_{0}$, then $\varphi_{e}^{\lambda}$ searches for a restriction on the possible values of the $e^{\text {th }}$ coordinate of sequences in FIX $_{\mathcal{G}}$ which extend $\alpha_{0}$. If no restriction is found, then $\varphi_{e}^{\lambda}$ diverges. On the other hand, if a $c \in[0,1]$ is found such that $\alpha(e) \neq c$ for every $\alpha \in$ Fix $_{\mathcal{G}}$ which extends $\alpha_{0}$, then $\varphi_{e}^{\lambda}$ converges to a representation of $c$. It is important to note that the search will depend only on $\alpha_{0}$. In other words, if the search is successful, then the same $c \in[0,1]$ is chosen for every $\beta$ extending $\alpha_{0}$ and-more to the point - every representation $\lambda$ of $\beta$.

Assume that the search succeeds in finding a restricted value $c \in[0,1]$. Let $\alpha \in \operatorname{Fix}_{\mathcal{G}}$ be an extension of $\alpha_{0}$. By the definition of $\Psi$ in Lemma 6.2, the fact that $\varphi_{e}^{\lambda}$ is a representation of $c$ for every representation $\lambda$ of $\alpha$ implies that $\beta(e)=c$ for every $\beta \in \Psi(\alpha)$. In particular, $\alpha \in \Psi(\alpha)$ implies $\alpha(e)=c$. But this contradicts the fact that $c$ cannot be the $e^{\text {th }}$ coordinate of any element of FIX F $_{\mathcal{G}}$ which extends $\alpha_{0}$. Therefore, the search for a restriction must fail; so for any $x \in[0,1]$, there is an extension of $\alpha_{0}$ to a fixed point $\alpha$ of $\Psi$ such that $\alpha \in \Pi[\mathcal{G}]$ and $\alpha(e)=x$. Therefore, $e \in \mathbb{N}$ satisfies the lemma.

We must now describe the details of the definition of $\varphi_{e}^{\lambda}$. For each $n \in \mathbb{N}$ such that $\alpha_{0}(n) \downarrow$, let $B_{n} \subseteq \mathbb{N}$ to be the binary representation of $\alpha_{0}(n)$. For concreteness, whenever $\alpha_{0}(n)$ is a binary rational we take $B_{n}$ to be finite. We can uniformly compute each of the sets $B_{n}$ from any representation $\lambda$ of any extension $\beta \in[0,1]^{\mathbb{N}}$ of $\alpha_{0}$. This is because it takes only a finite amount of information to record where $\alpha_{0}$ is defined, for which coordinates $n \in \mathbb{N}$ the value $\alpha_{0}(n)$ is a binary rational, and what each of these values is. The remainder of 
the construction is done relative to $B=\bigoplus\left\{B_{n} \mid \alpha_{0}(n) \downarrow\right\}$, guaranteeing that the result of the search will be independent of the choices of $\beta$ and $\lambda$.

Recall from the proof of Lemma 6.2 that $\{A \oplus D \subseteq \mathbb{N} \mid \Pi(D) \in \Psi(\Pi(A))\}$ is a $\Pi_{1}^{0}$ class. It should also be clear that $\{A \oplus Y \subseteq \mathbb{N} \mid \Pi(A)=\Pi(Y)\}$ is a $\Pi_{1}^{0}$ class. Using König's Lemma, it can be shown that the computable projection of a $\Pi_{1}^{0}$ class is again a $\Pi_{1}^{0}$ class $[5$, Theorem $9.9(c)]$ (note also that this is uniform and relativizes as expected). So we see that

$$
\{A \subseteq \mathbb{N} \mid \Pi(A) \in \mathcal{G}\}=\{A \subseteq \mathbb{N} \mid(\exists Y \subseteq \mathbb{N})[Y \in \mathcal{G} \text { and } \Pi(A)=\Pi(Y)]\}
$$

is a $\Pi_{1}^{0}$ class. Therefore, $\left\{A \subseteq \mathbb{N} \mid \Pi(A) \in \mathrm{FIX}_{\mathcal{G}}\right\}$ is a $\Pi_{1}^{0}$ class. Let $\mathbf{b}=\operatorname{deg}_{T}(B)$ and consider the $\Pi_{1}^{0}(\mathbf{b})$ class

$$
\left\{A \subseteq \mathbb{N} \mid \Pi(A) \text { extends } \alpha_{0}\right\}=\left\{A \subseteq \mathbb{N} \mid(\forall n)\left[\alpha_{0}(n) \downarrow \Longrightarrow \pi\left(A_{(n)}\right)=\pi\left(B_{n}\right)\right]\right\}
$$

where $A_{(n)}=\{i \mid\langle n, i\rangle \in A\}$ and $\pi: 2^{\mathbb{N}} \rightarrow[0,1]$ takes every binary representation to the real that it represents. So finally, for each $e \in \mathbb{N}$,

$$
\mathcal{F}_{e}=\left\{X \subseteq \mathbb{N} \mid(\exists A \subseteq \mathbb{N})\left[\Pi(A) \in \operatorname{FIX}_{\mathcal{G}}, \Pi(A) \text { extends } \alpha_{0} \text { and } A_{(e)}=X\right]\right\}
$$

is a $\Pi_{1}^{0}(\mathbf{b})$ class. Note that $\mathcal{F}_{e}$ is the class of binary expansions of reals $x \in[0,1]$ such that $\alpha_{0}$ can be extended to an $\alpha \in$ FiX $_{\mathcal{G}}$ with $\alpha(e)=x$. By the recursion theorem, there is an $e \in \mathbb{N}$ such that if $\lambda$ is a representation of an extension of $\alpha_{0}$, then $\varphi_{e}^{\lambda}$ builds the b-computable tree $T \subseteq 2^{<\mathbb{N}}$ associated to $\mathcal{F}_{e}$ and waits to find a $\sigma \in 2^{<\mathbb{N}}$ such that $\sigma \notin T$. When such a $\sigma$ is found, $\varphi_{e}^{\lambda}$ converges to a representation of $c=\pi\left(\sigma^{\widehat{\gamma}} 0^{\omega}\right) \in[0,1]$. This completes the definition of $\varphi_{e}^{\lambda}$. $\dashv$

We can now characterize the Turing ideals of non-total continuous degrees.

TheOREM 9.3. If $\mathcal{I}$ is a countable ideal in the Turing degrees, then $\mathcal{I}$ is a Scott ideal iff there is a non-total continuous degree $\mathbf{v}$ such that $\mathcal{I}=\mathcal{I}_{T}(\mathbf{v})$.

Proof. We have already observed that the ideal below a non-total continuous degree must be a Scott ideal, so only the other direction requires proof. Let $\mathcal{I}=\left\{\mathbf{v}_{\mathbf{i}}\right\}_{i \in \mathbb{N}}$ be a Scott ideal and let $\left\{x_{i}\right\}_{i \in \mathbb{N}}$ be a sequence of reals in $[0,1]$ with $\operatorname{deg}_{T}\left(x_{i}\right)=\mathbf{v}_{\mathbf{i}}$. We construct a sequence $\left\{\alpha_{s}\right\}_{s \in \mathbb{N}}$ of partial functions $\alpha_{s}: \mathbb{N} \rightarrow$ $[0,1]$ such that if $s<t$, then $\alpha_{t}$ is a proper extension of $\alpha_{s}$. We require that each $\alpha_{s}$ be defined on a finite initial segment of $\mathbb{N}$ and be extendable to a fixed point of $\Psi$. By Lemma 9.1, $\alpha=\bigcup_{s} \alpha_{s}$ is a fixed point of $\Psi$, so $\alpha$ is not computably diagonalizable and $\mathbf{v}=\operatorname{deg}_{r}(\alpha)$ is non-total. Additionally, we require that the reals in the range of $\alpha_{s}$ all have degree in $\mathcal{I}$ and that $x_{s} \in \operatorname{range}\left(\alpha_{s+1}\right)$. A sequence which is not computably diagonalizable lists exactly the reals in $[0,1]$ which it computes, so this ensures that $\mathcal{I}_{T}(\mathbf{v})=\mathcal{I}$.

The Construction.

Stage $s=0$. Let $\alpha_{0}=\emptyset$.

Stage $s+1$. (We ensure that $x_{s} \in \operatorname{range}(\alpha)$ ) ) Assume that we have defined $\alpha_{s}$. Let $e$ be the coordinate guaranteed by Lemma 9.2 with $\mathcal{G}=2^{\mathbb{N}}$. Define a 
partial sequence

$$
\alpha_{s}^{\prime}(i)= \begin{cases}\alpha_{s}(i), & \text { if } i \in \operatorname{range}\left(\alpha_{s}\right) \\ x_{s}, & \text { if } i=e .\end{cases}
$$

Hence, $\alpha_{s}^{\prime}$ can be extended to a fixed point of $\Psi$. The reals in the range of $\alpha_{s}^{\prime}$ have degree in $\mathcal{I}$, so there is an $\mathbf{b} \in \mathcal{I}$ which computes $\alpha_{s}^{\prime}$. Take $\mathbf{c} \in \mathcal{I}$ such that $\mathbf{c} \gg \mathbf{b}$. Then $\mathbf{c}$ computes a fixed point $\widehat{\alpha}$ of $\Psi$ extending $\alpha_{s}^{\prime}$ because $\left\{A \subseteq \mathbb{N} \mid \widehat{\alpha}=\Pi(A)\right.$ extends $\alpha_{s}^{\prime}$ and $\left.\widehat{\alpha} \in \Psi(\widehat{\alpha})\right\}$ is a $\Pi_{1}^{0}(\mathbf{b})$ class. Every real in the range of $\widehat{\alpha}$ is computable from c, so each must have degree in $\mathcal{I}$. Let $\alpha_{s+1}=\widehat{\alpha} \uparrow(e+1)$ and note that $\alpha_{s+1}$ satisfies all of our requirements.

This completes the construction and the proof.

Corollary 9.4. The class $\mathcal{S} \subseteq 2^{\mathbb{N}}$ is a Scott set iff $\mathcal{S}=\left\{A \subseteq \mathbb{N} \mid A \leq_{r} f\right\}$ for some $f \in \mathcal{C}[0,1]$ of non-total degree.

We can combine the method used in the proof above with constructions from classical computability theory to derive results about the continuous degrees which share a given Turing ideal. For the first example, we build a full binary tree of partial sequences to prove that every Scott ideal is the Turing ideal below continuum many incomparable continuous degrees. Compare this to Corollary 4.5 , which says that a continuous degree is uniquely determined by the class of total degrees above it.

Theorem 9.5. If $\mathcal{I}$ is a Scott ideal in the Turing degrees, then there are $2^{\aleph_{0}}$ pairwise incomparable continuous degrees with Turing ideal $\mathcal{I}$.

Proof. Let $\left\{x_{i}\right\}_{i \in \mathbb{N}}$ be a sequence of reals from $[0,1]$ representing the degrees in $\mathcal{I}$. For each $\sigma \in 2^{<\mathbb{N}}$, we construct a partial function $\alpha_{\sigma}: \mathbb{N} \rightarrow[0,1]$ such that if $\sigma \subset \tau$, then $\alpha_{\tau}$ is a proper extension of $\alpha_{\sigma}$. As in the previous proof, we require that each $\alpha_{\sigma}$ be defined on a finite initial segment of $\mathbb{N}$, that each can be extended to a fixed point of $\Psi$, that the reals in the range of $\alpha_{\sigma}$ all have degree in $\mathcal{I}$ and that $x_{|\sigma|-1} \in \operatorname{range}\left(\alpha_{\sigma}\right)$ (when $\sigma \neq \emptyset$ ). If $f \in 2^{\mathbb{N}}$, let $\alpha_{f}=\bigcup_{\sigma \subset f} \alpha_{\sigma}$. As before, the requirements placed on $\left\{\alpha_{\sigma}\right\}_{\sigma \in 2<\mathbb{N}}$ guarantee that $\alpha_{f}$ is not computably diagonalizable and $\mathcal{I}_{T}\left(\alpha_{f}\right)=\mathcal{I}$. We also want to ensure that if $f, g \in 2^{\mathbb{N}}$ and $f \neq g$, then $\left.\alpha_{f}\right|_{r} \alpha_{g}$. This is done by meeting, for each $e \in \mathbb{N}$, the following requirement.

$$
\begin{aligned}
R_{e}: & \text { For every } \sigma, \tau \in 2^{e+1} \text { with } \sigma \neq \tau, \text { if } \gamma_{\sigma} \text { and } \gamma_{\tau} \text { are any } \\
& \text { total extensions of } \alpha_{\sigma} \text { and } \alpha_{\tau} \text { to fixed points of } \Psi \text {, then } \\
& \text { there is a representation } \lambda \text { of } \gamma_{\sigma} \text { such that } \varphi_{e}^{\lambda} \text { does not } \\
& \text { represent } \gamma_{\tau} .
\end{aligned}
$$

\section{The Construction.}

Stage $s=0$. Let $\alpha_{\emptyset}=\emptyset$.

Stage $s+1$. We are given $\alpha_{\sigma}$, for each $\sigma \in 2^{s}$.

Step 1. (We ensure that $x_{s} \in \operatorname{range}\left(\alpha_{f}\right)$, for each $f \in 2^{\mathbb{N}}$.) For each $\sigma \in 2^{s}$, we can use Lemma 9.2 to extend $\alpha_{\sigma}$ to a partial sequence $\beta_{\sigma}$ such that $x_{s} \in$ 
range $\left(\beta_{\sigma}\right)$, as in the proof of the previous theorem. Of course, $\beta_{\sigma}$ must meet the same requirements as $\alpha_{\sigma}$. In particular, $\beta_{\sigma}$ is defined on a finite initial segment of $\mathbb{N}$, it can be extended to a fixed point of $\Psi$, and the reals in its range have degrees in $\mathcal{I}$.

Step 2. (We satisfy $R_{s}$.) For each $\rho \in 2^{s}$, we temporarily set $\alpha_{\rho \wedge}=$ $\alpha_{\rho \wedge 1}=\beta_{\rho}$; these will be redefined to satisfy $R_{s}$. Take $\sigma, \tau \in 2^{s+1}$ with $\sigma \neq \tau$. Let $\gamma_{\sigma} \in[0,1]^{\mathbb{N}}$ be an extension of $\alpha_{\sigma}$ to a fixed point of $\Psi$ and let $\lambda$ be a representation of $\gamma_{\sigma}$ such that $\varphi_{s}^{\lambda}$ represents an extension $\gamma_{\tau}$ of $\alpha_{\tau}$. If no such $\gamma_{\sigma}, \gamma_{\tau}$ and $\lambda$ exist, then $R_{s}$ is already satisfied for $\sigma$ and $\tau$. So we may assume, for the sake of argument, that they have been given.

Let $e \in \mathbb{N}$ be the coordinate provided by Lemma 9.2 applied to $\alpha_{\tau}$ (again with $\left.\mathcal{G}=2^{\mathbb{N}}\right)$. Extend $\alpha_{\tau}$ to $\alpha_{\tau}^{\prime}$ so that $\alpha_{\tau}^{\prime}(e) \neq \gamma_{\tau}(e)$. As usual, ensure that $\alpha_{\tau}^{\prime}$ is defined on a finite initial segment of $\mathbb{N}$, extends to a fixed point of $\Psi$, and that all reals in the range have degree in $\mathcal{I}$. We now show how to extend $\alpha_{\sigma}$. Take $\varepsilon_{0} \in \mathbb{Q}^{+}$such that $\varepsilon_{0} \leq 2^{e}\left|\alpha_{\tau}^{\prime}(e)-\gamma_{\tau}(e)\right|$ and let $F \subseteq \mathbb{Q}^{+}$be the finite use of the computation of $\varphi_{s}^{\lambda}\left(\varepsilon_{0}\right)$. Then for any $\lambda_{0}$ which agrees with $\lambda$ on $F, \varphi_{s}^{\lambda_{0}}$ cannot represent an extension of $\alpha_{\tau}^{\prime}$. Choose $\delta \in \mathbb{Q}^{+}$small enough that for every $\varepsilon \in F$, $d_{[0,1]^{\mathbb{N}}}\left(\gamma_{\sigma}-\lambda(\varepsilon)\right) \leq \varepsilon-\delta$. Choose $\mathbf{a} \mathbf{b} \in \mathcal{I}$ which computes $\alpha_{\sigma}$ and consider the $\Pi_{1}^{0}(\mathbf{b})$ class

$$
\begin{aligned}
\mathcal{G}=\{A \subseteq \mathbb{N} \mid & \Pi(A) \text { extends } \alpha_{\sigma} \text { to a fixed point of } \Psi \\
& \text { and } \left.(\forall \varepsilon \in F)\left[d_{[0,1]^{\mathbb{N}}}(\Pi(A)-\lambda(\varepsilon)) \leq \varepsilon-\delta\right]\right\} .
\end{aligned}
$$

Because $\gamma_{\sigma} \in \Pi[\mathcal{G}]$, we know that $\mathcal{G}$ is nonempty. Take $\mathbf{c} \in \mathcal{I}$ such that $\mathbf{c} \gg \mathbf{b}$. Then c computes an element of $\mathcal{G}$, so c computes an extension $\widehat{\gamma}_{\sigma}$ of $\alpha_{\sigma}$ to a fixed point of $\Psi$ such that $\widehat{\gamma}_{\sigma}$ has a representation $\lambda_{0}$ which agrees with $\lambda$ on $F$. Choose $m \in \mathbb{N}$ large enough that $\alpha_{\sigma}^{\prime}=\widehat{\gamma}_{\sigma} \uparrow m$ extends $\alpha_{\sigma}$ and every total extension of $\alpha_{\sigma}^{\prime}$ has a representation which agrees with $\lambda$ on $F$. Every real in the range of $\alpha_{\sigma}^{\prime}$ is computable from $\mathbf{c}$, so each must have degree in $\mathcal{I}$. By redefining $\alpha_{\sigma}$ and $\alpha_{\tau}$ to be $\alpha_{\sigma}^{\prime}$ and $\alpha_{\tau}^{\prime}$, we ensure that $R_{s}$ is satisfied for the current choice of $\sigma$ and $\tau$. Repeat the argument until $R_{s}$ has been satisfied for every ordered pair of distinct strings $\sigma, \tau \in 2^{s+1}$.

COROLlary 9.6. There are continuous functions $f, g \in \mathcal{C}[0,1]$ such that $\left.f\right|_{r} g$ and $f$ and $g$ compute the same subsets of $\mathbb{N}$.

The final theorem allows us to strengthen the corollary by fixing one of the functions. The proof uses a slight variation on the technique from [12] of forcing with $\Pi_{1}^{0}$ classes. Note also that this proof uses the full strength of Lemma 9.2, unlike the other proofs in this section.

TheOREM 9.7. For any non-total continuous degree $\mathbf{v}$, there is a continuous degree $\mathbf{w}$ such that $\mathbf{v} \mid \mathbf{w}$ and $\mathcal{I}_{T}(\mathbf{v})=\mathcal{I}_{T}(\mathbf{w})$.

Proof. Let $\alpha \in[0,1]^{\mathbb{N}}$ be a sequence of degree $\mathbf{v}$. We construct a sequence $\left\{\beta_{s}\right\}_{s \in \mathbb{N}}$ of partial functions $\beta_{s}: \mathbb{N} \rightarrow[0,1]$ such that if $s<t$, then $\beta_{t}$ is a proper extension of $\beta_{s}$. At the same time, we construct a sequence $\left\{\mathcal{G}_{s}\right\}_{s \in \mathbb{N}}$ of $\Pi_{1}^{0}$ classes 
$2^{\mathbb{N}}=\mathcal{G}_{0} \supseteq \mathcal{G}_{1} \supseteq \mathcal{G}_{2} \supseteq \cdots$ such that each $\beta_{s}$ can be extended to a fixed point of $\Psi$ in $\Pi\left[\mathcal{G}_{s}\right]$. Furthermore, we require that each $\beta_{s}$ be defined on a finite initial segment of $\mathbb{N}$, that range $\left(\beta_{s}\right) \subseteq \operatorname{range}(\alpha)$ and that $\alpha(s) \in \operatorname{range}\left(\beta_{s+1}\right)$. Let $\beta=\bigcup_{s} \beta_{s} \in[0,1]^{\mathbb{N}}$ and $\mathbf{w}=\operatorname{deg}_{T}(\beta)$. The requirements guarantee that $\beta$ is not computably diagonalizable and, because $\alpha$ and $\beta$ list the same reals, that $\mathcal{I}_{T}(\mathbf{v})=\mathcal{I}_{T}(\mathbf{w})$. Also note that $\beta \in \Pi\left[\mathcal{G}_{s}\right]$, for each $s \in \mathbb{N}$. Finally, to ensure that $\left.\alpha\right|_{r} \beta$, we meet the following requirements for each $e \in \mathbb{N}$.

\section{$R_{e}$ : There is a representation $\lambda$ of $\alpha$ such that $\varphi_{e}^{\lambda}$ does not represent any extension of $\beta_{e+1}$. \\ $S_{e}$ : For every extension $\gamma \in \Pi\left[\mathcal{G}_{e+1}\right]$ of $\beta_{e+1}$ to a fixed point of $\Psi$, there is a representation $\lambda$ of $\gamma$ such that $\varphi_{e}^{\lambda}$ does not represent $\alpha$.}

\section{The Construction.}

Stage $s=0$. Let $\beta_{0}=\emptyset$ and $\mathcal{G}_{0}=2^{\mathbb{N}}$.

Stage $s+1$. Assume that we have defined a partial sequence $\beta_{s}$ and a $\Pi_{1}^{0}$ class $\mathcal{G}_{s} \subseteq 2^{\mathbb{N}}$ satisfying the requirements given. In particular, $\beta_{s}$ can be extended to a fixed point of $\Psi$ in $\Pi\left[\mathcal{G}_{s}\right]$.

Step 1. (We ensure that $\alpha(s) \in \operatorname{range}\left(\beta_{s+1}\right)$ ) ) Let $e_{1}$ be the coordinate guaranteed by Lemma 9.2 for $\beta_{s}$ and $\mathcal{G}_{s}$. Define a partial sequence

$$
\beta_{s}^{\prime}(i)= \begin{cases}\beta_{s}(i), & \text { if } i \in \operatorname{range}\left(\beta_{s}\right) \\ \alpha(s), & \text { if } i=e_{1} .\end{cases}
$$

Because $\beta_{s+1}$ will extend $\beta_{s}^{\prime}$, we have ensured that $\alpha(s) \in \operatorname{range}\left(\beta_{s+1}\right)$. We also know that $\beta_{s}^{\prime}$ extends to a fixed point of $\Psi$ in $\Pi\left[\mathcal{G}_{s}\right]$.

Step 2. (We satisfy $R_{s}$.) Now apply Lemma 9.2 to $\beta_{s}^{\prime}$ and $\mathcal{G}_{s}$ to get a new coordinate $e_{2}$. Let $\lambda$ be any representation of $\alpha$. If $\varphi_{s}^{\lambda}$ is not a representation of a sequence $\gamma \in[0,1]^{\mathbb{N}}$, then set $\beta_{s}^{\prime \prime}=\beta_{s}^{\prime}$. Otherwise, extend $\beta_{s}^{\prime}$ to a partial sequence $\beta_{s}^{\prime \prime}$ by setting $\beta_{s}^{\prime \prime}\left(e_{2}\right)$ equal to any real in range $(\alpha) \backslash\left\{\gamma\left(e_{2}\right)\right\}$. Either way, $\varphi_{s}^{\lambda}$ does not represent any extension of $\beta_{s}^{\prime \prime}$. But $\beta_{s+1}$ will extend $\beta_{s}^{\prime \prime}$, so $R_{s}$ will be satisfied. Of course, $\beta_{s}^{\prime \prime}$ can be extended to a fixed point of $\Psi$ in $\Pi\left[\mathcal{G}_{s}\right]$ and range $\left(\beta_{s}^{\prime \prime}\right) \subseteq \operatorname{range}(\alpha)$.

Step 3. (We satisfy $S_{s}$.) This is the heart of the proof. Recall from Lemma 6.6 that there is a computable function $\Gamma: 2^{\mathbb{N}} \rightarrow \mathbb{N}^{\mathbb{Q}^{+}}$taking every b-representation to a representation of the same sequence. For each $\varepsilon \in \mathbb{Q}^{+}$, consider the $\Pi_{1}^{0}$ class $\mathcal{F}_{\varepsilon}=\left\{A \subseteq \mathcal{G}_{s} \mid \varphi_{s}^{\Gamma(A)}(\varepsilon) \uparrow\right\} \subseteq \mathcal{G}_{s}$. The proof breaks into two cases, based on whether or not there exists an $\varepsilon \in \mathbb{Q}^{+}$such that $\beta_{s}^{\prime \prime}$ can be extended to a fixed point of $\Psi$ which is in $\Pi\left[\mathcal{F}_{\varepsilon}\right]$.

First assume that there is such an $\varepsilon$. As in previous arguments, the brepresentations of fixed points of $\Psi$ which extend $\beta_{s}^{\prime \prime}$ form a $\Pi_{1}^{0}(\mathbf{b})$ class, where $\mathbf{b}$ is the Turing degree of $\beta_{s}^{\prime \prime}$. We have assumed that the intersection of this class with $\mathcal{F}_{\varepsilon}$ is nonempty. By Theorem 8.4, there is a total degree $\mathbf{c}$ such that 
$\mathbf{b} \ll \mathbf{c}<\mathbf{v}$, so $\alpha$ computes an element from any nonempty $\Pi_{1}^{0}(\mathbf{b})$ class. Therefore, $\alpha$ computes (a b-representation of) an extension $\gamma \in \Pi\left[\mathcal{F}_{\varepsilon}\right]$ of $\beta_{s}^{\prime \prime}$ to a fixed point of $\Psi$. Let $\mathcal{G}_{s+1}=\mathcal{F}_{\varepsilon}$ and $\beta_{s+1}=\gamma \uparrow n$, where $n=1+\max \left\{i \mid \beta_{s}^{\prime \prime}(i) \downarrow\right\}$. This satisfies requirement $S_{s}$, because every extension $\gamma \in \Pi\left[\mathcal{G}_{s+1}\right]$ of $\beta_{s+1}$ to a fixed point of $\Psi$ has a representation $\lambda$ for which $\varphi_{s}^{\lambda}(\varepsilon) \uparrow$. Because $\gamma$ is computable from $\alpha$, range $(\gamma) \subseteq \operatorname{range}(\alpha)$, so range $\left(\beta_{s+1}\right) \subseteq \operatorname{range}(\alpha)$ as is required.

Now assume that for every $\varepsilon \in \mathbb{Q}^{+}$, no extension of $\beta_{s}^{\prime \prime}$ to a fixed point of $\Psi$ is in $\Pi\left[\mathcal{F}_{\varepsilon}\right]$. As above, $\alpha$ computes a b-representation $A \subseteq \mathbb{N}$ of an extension $\gamma \in \Pi\left[\mathcal{G}_{s}\right]$ of $\beta_{s}^{\prime \prime}$ to a fixed point of $\Psi$. Since $A$ cannot also compute $\alpha$-or $\alpha \equiv_{r} A$ would have total degree $-\varphi_{s}^{\Gamma(A)}$ is not a representation of $\alpha$. But we know that $\varphi_{s}^{\Gamma(A)}(\varepsilon) \downarrow$, for every $\varepsilon \in \mathbb{Q}^{+}$, because $\gamma \notin \Pi\left[\mathcal{F}_{\varepsilon}\right]$. Therefore, there is an $\varepsilon$ such that $d_{[0,1]^{\mathbb{N}}}\left(\alpha, \varphi_{s}^{\Gamma(A)}(\varepsilon)\right) \geq \varepsilon$. Choose $m \in \mathbb{N}$ large enough so that $\varphi_{s}^{\Gamma(A\lceil m)}(\varepsilon) \downarrow$. Now choose $n \in \mathbb{N}$ large enough so that $\beta_{s+1}=\gamma \uparrow n$ extends $\beta_{s}^{\prime \prime}$ and every total extension of $\beta_{s+1}$ has a b-representation extending $A \uparrow m$. This implies that every total extension of $\beta_{s+1}$ has a representation $\lambda$ such that $\varphi_{s}^{\lambda}(\varepsilon)=$ $\varphi_{s}^{\Gamma(A)}(\varepsilon)$, hence $\varphi_{s}^{\lambda}$ is not a representation of $\alpha$. Therefore, $S_{s}$ is satisfied without even specifying $\mathcal{G}_{s+1}$ (so just take $\mathcal{G}_{s+1}=\mathcal{G}_{s}$ ). Note again that $\operatorname{range}\left(\beta_{s+1}\right) \subseteq$ range $(\alpha)$.

This completes the construction and the proof.

Corollary 9.8. For any $f \in \mathcal{C}[0,1]$ of non-total degree, there is a $g \in \mathcal{C}[0,1]$ such that $\left.f\right|_{r} g$ and $f$ and $g$ compute the same subsets of $\mathbb{N}$.

Acknowledgments. The author is grateful to Steffen Lempp, who introduced him to Question 1.2, and to the many people with whom he has discussed the problem and its solution.

\section{REFERENCES}

[1] M. M. Arslanov, R. F. Nadyrov, and V. D. Solov'Ev, A criterion for the completeness of recursively enumerable sets, and some generalizations of a fixed point theorem, Izvestija Vysših Učebnyh Zavedeniı Matematika, (1977), no. 4 (179), pp. 3-7.

[2] Michael J. BeEson, Foundations of constructive mathematics, Ergebnisse der Mathematik und ihrer Grenzgebiete (3) [Results in Mathematics and Related Areas (3)], vol. 6, SpringerVerlag, Berlin, 1985, Metamathematical studies.

[3] G. D. Birkhoff and O. D. KellogG, Invariant points in function space, Transactions of the American Mathematical Society, vol. 23 (1922), no. 1, pp. 96-115.

[4] H. F. Bohnenblust and S. Karlin, On a theorem of Ville, Contributions to the theory of games, Annals of Mathematics Studies, no. 24, Princeton University Press, Princeton, N.J., 1950, pp. $155-160$.

[5] D. Cenzer and J. B. Remmel, $\Pi_{1}^{0}$ classes in mathematics, Handbook of recursive mathematics, vol. 2, Studies in Logic and the Foundations of Mathematics, vol. 139, North-Holland, Amsterdam, 1998, pp. 623-821.

[6] Douglas Cenzer, $\Pi_{1}^{0}$ classes in computability theory, Handbook of computability theory, Studies in Logic and the Foundations of Mathematics, vol. 140, North-Holland, Amsterdam, 1999, pp. 37-85.

[7] S. B. Cooper, Partial degrees and the density problem, The Journal of Symbolic Logic, vol. 47 (1982), no. 4, pp. 854-859 (1983). 
[8] Samuel Eilenberg and Deane Montgomery, Fixed point theorems for multi-valued transformations, American Journal of Mathematics, vol. 68 (1946), pp. 214-222.

[9] Andrzej Grzegorczyk, Computable functionals, Fundamenta Mathematicae, vol. 42 (1955), pp. 168-202.

[10] - On the definitions of computable real continuous functions, Fundamenta Mathematicae, vol. 44 (1957), pp. 61-71.

[11] Lance Gutteridge, Some results on enumeration reducibility, Ph.D. Dissertation, Simon Fraser University, 1971.

[12] Carl G. Jockusch, JR. and Robert I. Soare, $\Pi_{1}^{0}$ classes and degrees of theories, Transactions of the American Mathematical Society, vol. 173 (1972), pp. 33-56.

[13] Shizuo Kakutani, A generalization of Brouwer's fixed point theorem, Duke Mathematical Journal, vol. 8 (1941), pp. 457-459.

[14] Stephen Cole Kleene, Introduction to metamathematics, D. Van Nostrand Co., Inc., New York, N. Y., 1952.

[15] Christoph Kreitz and Klaus Weinrauch, Theory of representations, Theoretical Computer Science, vol. 38 (1985), no. 1, pp. 35-53.

[16] Daniel Lacombe, Extension de la notion de fonction récursive aux fonctions d'une ou plusieurs variables réelles. I, Comptes Rendus Mathématique. Académie des Sciences. Paris, vol. 240 (1955), pp. 2478-2480.

[17] _ Extension de la notion de fonction récursive aux fonctions d'une ou plusieurs variables réelles. II, III, Comptes Rendus Mathématique. Académie des Sciences. Paris, vol. 241 (1955), pp. 13-14, 151-153.

[18] - Quelques procédés de définition en topologie recursive, Constructivity in mathematics: Proceedings of the colloquium held at Amsterdam, 1957 (edited by A. Heyting), Studies in Logic and the Foundations of Mathematics, North-Holland Publishing Co., 1959, pp. 129-158.

[19] Manuel Lerman, Degrees of unsolvability, Perspectives in Mathematical Logic, SpringerVerlag, Berlin, 1983, Local and global theory.

[20] Yu. T. Medvedev, Degrees of difficulty of the mass problem, Doklady Akademii Nauk $\boldsymbol{S S S R}$, vol. 104 (1955), pp. 501-504.

[21] A. A. MUČnik, On strong and weak reducibility of algorithmic problems, Akademija Nauk SSSR. Sibirskoe Otdelenie. Sibirskiǔ Matematičeskiǔ Žurnal, vol. 4 (1963), pp. 1328-1341.

[22] John Myhill, Note on degrees of partial functions, Proceedings of the American Mathematical Society, vol. 12 (1961), pp. 519-521.

[23] Anil Nerode and Richard A. Shore, Second order logic and first order theories of reducibility orderings, The Kleene Symposium (Proc. Sympos., Univ. Wisconsin, Madison, Wis., 1978), Stud. Logic Foundations Math., vol. 101, North-Holland, Amsterdam, 1980, pp. 181-200.

[24] V. P. ORevkov, A constructive map of the square into itself, which moves every constructive point, Doklady Akademii Nauk SSSR, vol. 152 (1963), pp. 55-58.

[25] David B. Posner, The upper semilattice of degrees $\leq \mathbf{0}^{\prime}$ is complemented, J. Symbolic Logic, vol. 46 (1981), no. 4, pp. 705-713.

[26] Marian B. Pour-El and Jerome Caldwell, On a simple definition of computable function of a real variable - with applications to functions of a complex variable, Zeitschrift für Mathematische Logik und Grundlagen der Mathematik, vol. 21 (1975), pp. 1-19.

[27] Marian B. Pour-El and J. IAn Richards, Computability and noncomputability in classical analysis, Transactions of the American Mathematical Society, vol. 275 (1983), no. 2, pp. 539560 .

[28] - Computability in analysis and physics, Springer-Verlag, Berlin, 1989.

[29] Linda Jean Richter, Degrees of structures, Ph.D. Dissertation, University of Illinois at Urbana-Champaign, 1979.

[30] HARTLEy Rogers, JR., Theory of recursive functions and effective computability, McGraw-Hill Book Co., New York, 1967.

[31] M. RozInas, The semilattice of e-degrees, Recursive functions (Russian), Ivanov. Gos. Univ., Ivanovo, 1978, pp. 71-84.

[32] Gerald E. SaCKs, The recursively enumerable degrees are dense, Annals of Mathematics. Second Series, vol. 80 (1964), pp. 300-312. 
[33] J. Schauder, Der Fixpunktsatz in Funktionalräumen, Studia Mathematica, vol. 2 (1930), pp. $171-180$.

[34] Dana ScotT, Algebras of sets binumerable in complete extensions of arithmetic, Proceedings of symposia in pure mathematics, vol. v, American Mathematical Society, Providence, R.I., 1962, pp. $117-121$.

[35] Alan L. Selman, Arithmetical reducibilities. I, Zeitschrift für Mathematische Logik und Grundlagen der Mathematik, vol. 17 (1971), pp. 335-350.

[36] Stephen G. Simpson, Degrees of unsolvability: A survey of results, Handbook of mathematical logic (J. Barwise, editor), North-Holland, Amsterdam, 1977, pp. 631-652.

[37] - First-order theory of the degrees of recursive unsolvability, Annals of Mathematics. Second Series, vol. 105 (1977), no. 1, pp. 121-139.

[38] Theodore A. Slaman and W. Hugh Woodin, Definability in the enumeration degrees, Archive for Mathematical Logic, vol. 36 (1997), no. 4-5, pp. 255-267, Sacks Symposium (Cambridge, MA, 1993).

[39] Clifford Spector, On degrees of recursive unsolvability, Annals of Mathematics. Second Series, vol. 64 (1956), pp. 581-592.

[40] A. M. TURING, On computable numbers, with an application to the Entscheidungsproblem, Proceedings of the London Mathematical Society. Second Series, vol. 42 (1936), pp. 230-265, Correction in [41].

[41] - On computable numbers, with an application to the Entscheidungsproblem. A correction, Proceedings of the London Mathematical Society. Second Series, vol. 43 (1937), pp. 544546 , Correction to [40].

[42] Klaus Weihrauch, Computability on computable metric spaces, Theoretical Computer Science, vol. 113 (1993), no. 2, pp. 191-210.

[43] — Computable analysis, an introduction, Springer-Verlag, Berlin, 2000.

\author{
DEPARTMENT OF MATHEMATICS \\ INDIANA UNIVERSITY \\ BLOOMINGTON, IN 47405-5701, USA \\ E-mail: millerj7@indiana.edu
}

